\title{
Comparative transcriptomic analysis reveals potential mechanisms for high tolerance to submergence in arbor willows
}

\author{
Yanhong Chen Equal first author, 1, 2, Jie Yang ${ }^{\text {Equal first author, 1,2 }}$, Hongyi Guo ${ }^{1,2}$, Yawen Du ${ }^{1,2}$, Guoyuan Liu ${ }^{1,2}$, Chunmei Yu ${ }^{1,2}$, Fei \\ Zhong $^{1,2}$, Bolin Lian ${ }^{1,2}$, Jian Zhang ${ }^{\text {Corresp. } 1,2}$ \\ ${ }^{1}$ School of Life Science, Nantong University, Nantong, Jiangsu Province, China \\ 2 Key Lab of Landscape Plant Genetics and Breeding, Nantong, China \\ Corresponding Author: Jian Zhang \\ Email address: ntdxylzw@163.com
}

Background. Submergence threatens plant growth and survival by decreasing or eliminating oxygen supply. Uncovering the complex regulatory network underlying the tolerance of Salix to submergence and identifying the key regulators are important for molecular-assisted breeding of Salix. Methods. In this study, we screened germplasm resources of arbor willows and discovered both submergence-tolerant and -sensitive varieties. Then, by performing RNA-seq, we compared the differences between the transcriptomes of two varieties, i.e., the submergence-tolerant variety "Suliu 795" and the submergence-sensitive variety "Yanliu No. 1," and the different submergence treatment time points to identify the potential mechanisms of submergence in Salix and the unique approaches by which the variety "Suliu 795" possessed a higher tolerance compared to "Yanliu No. 1". Results. A total of 22790 differentially expressed genes were identified from 25 comparisons. Using gene ontology annotation and pathway enrichment analysis, the expression pattern of transcriptional factors, important players in hormone signaling, carbohydrate metabolism, and the anaerobic respiration pathway were found to differ significantly between the two varieties. The principal component analysis and qRT-PCR results verified the reliability of the RNA sequencing data. The results of further analysis indicated that "Suliu 795" had higher submergence tolerant activity than "Yanliu No. 1" because of three characteristics: (1) high sensitivity to the probable low oxygen stress and initiation of appropriate responding mechanisms in advance; (2) maintenance of energy homeostasis to prevent energy depletion under hypoxic stress; and (3) keep "quiescence" through fine-tuning the equilibrium between phytohormones GA, SA and ethylene. 
1 Comparative transcriptomic analysis reveals potential

2 mechanisms for high tolerance to submergence in

3 arbor willows

4 Yanhong Chen ${ }^{1,2 \dagger}$, Jie Yang ${ }^{1,2 \dagger}$, Hongyi Guo ${ }^{1,2}$, Yawen $\mathrm{Du}^{1,2}$, Guoyuan Liu ${ }^{1,2}$, Chunmei Yu ${ }^{1,2}$,

5 Fei Zhong ${ }^{1,2}$, Bolin Lian ${ }^{1,2}$, Jian Zhang ${ }^{1,2}$

$7 \quad{ }^{1}$ School of Life Science, Nantong University, Nantong, Jiangsu Province, China

$8{ }^{2}$ Key Lab of Landscape Plant Genetics and Breeding, Nantong, Jiangsu Province, China

9 †These authors contribute equally to this work

10

11 Corresponding Author:

12 Jian Zhang

13 E-mail: ntdxylzw@,163.com

14 
16

\section{Abstract}

Background. Submergence threatens plant growth and survival by decreasing or eliminating oxygen supply. Uncovering the complex regulatory network underlying the tolerance of Salix to submergence and identifying the key regulators are important for molecular-assisted breeding of Salix.

Methods. In this study, we screened germplasm resources of arbor willows and discovered both submergence-tolerant and -sensitive varieties. Then, by performing RNA-seq, we compared the differences between the transcriptomes of two varieties, i.e., the submergence-tolerant variety "Suliu 795" and the submergence-sensitive variety "Yanliu No. 1," and the different submergence treatment time points to identify the potential mechanisms of submergence in Salix and the unique approaches by which the variety "Suliu 795" possessed a higher tolerance compared to "Yanliu No. 1".

Results. A total of 22790 differentially expressed genes were identified from 25 comparisons. Using gene ontology annotation and pathway enrichment analysis, the expression pattern of transcriptional factors, important players in hormone signaling, carbohydrate metabolism, and the anaerobic respiration pathway were found to differ significantly between the two varieties. The principal component analysis and qRT-PCR results verified the reliability of the RNA sequencing data. The results of further analysis indicated that "Suliu 795" had higher submergence tolerant activity than "Yanliu No. 1" because of three characteristics: (1) high sensitivity to the probable low oxygen stress and initiation of appropriate responding mechanisms in advance; (2) maintenance of energy homeostasis to prevent energy depletion under hypoxic stress; and (3) keep "quiescence" through fine-tuning the equilibrium between phytohormones GA, SA and ethylene..

Keywords: submergence stress; hypoxia; Salix; transcriptome analysis; hormone; transcription factors; anaerobic respiration pathway 


\section{Introduction}

Water plays a central role in the interaction between plants and the environment. However, excess water can result in flooding, which negatively affects the developmental periods of plant lifecycle, from seed germination to vegetative and reproductive growth (Zhou et al., 2020). . Flooding can be classified as waterlogging or submergence (Fukao et al., 2019). Waterlogging occurs when the roots of plants are submerged in water and the soil is saturated, while submergence occurs when the aerial plant tissues are fully submerged in water (Sasidharan et al., 2017). Water surrounding roots or whole plants disrupts the exchange of oxygen between the plant and the atmosphere, leading to low oxygen availability (hypoxia $<21 \% \mathrm{O}_{2}$ ) or even complete absence of oxygen (anoxia) in plant tissue, which is detrimental to plant survival (Loreti al., 2016). Wetland plant species such as Juncus effuses have developed constitutive aerenchyma anatomical structures to facilitate gas diffusion between roots and the aerial environment (Fukao \& Bailey-Serres 2008). In crops such as maize, aerenchyma development can be induced by flooding or hypoxia (Visser \& Bögemann 2006). Another study reported a flood-adapted structure/organ that improves oxygen supply in plants during flooding via adventitious roots (Sauter 2013). During flooding or submergence, flooding-tolerant herbaceous plants and trees produce shoot-borne adventitious roots, as seen in the perennial wetland species Cotula coronopifolia and Meionectes brownie (Calvo-Polanco et al., 2012; Rich et al., 2012; Sauter 2013). Adventitious roots replace the sediment root system to improve plant growth by shortening the distance between the source of oxygen supply and oxygen consumer (cells, tissues, and organs). In addition, adventitious roots can reduce radial oxygen loss because of poorly developed endodermis or produce oxygen in situ (Calvo-Polanco et al., 2012; Rich et al., 2012; Sauter 2013).

A complex regulatory network in plant submergence or waterlogging tolerance has been revealed in model plants, such as Arabidopsis and rice, in which metabolic regulation pathways and phytohormone signaling pathways are interconnected (Fukao et al., 2006; Xu et al., 2006; Hattori et al., 2009; Licausi et al., 2010; Niroula et al., 2012; Voesenek \& Bailey-Serres 2015; Fukao et al., 2019). Upon submergence or waterlogging stress, the depletion of oxygen triggers the anaerobic respiration metabolism pathway (Voesenek \& Bailey-Serres 2015; Fukao et al., 2019). Sugar metabolism enzymes, such as alcohol dehydrogenase (ADH), pyruvate decarboxylase (PDC), and sucrose synthase (SUS) are significantly upregulated during waterlogging (Ismond et al., 2003; Kato-Noguchi \& Morokuma 2007). Gibberillic acids (GA), ethylene, auxin, and ABA are major phytohormones involved in submergence or waterlogging tolerance by promoting the formation of adventitious roots and aerenchyma and regulating the secondary metabolism pathway or plant growth, such as shoot elongation (Jackson 2008; Voesenek \& Bailey-Serres 2015; Qi et al., 2020). Flood-tolerant plants have developed many survival strategies, including development of adventitious roots and aerenchyma, the "escape" (low- $\mathrm{O}_{2}$ escape syndrome, LOES) and "quiescence" (low- $\mathrm{O}_{2}$ quiescence syndrome, LOQS) 
responses (Fukao \& Bailey-Serres 2008; Hattori et al., 2009; Akman et al., 2012; Niroula et al., 2012; Voesenek \& Bailey-Serres 2015). LOES and LOQS are two extreme strategies. The key regulators in "escape" or "quiescence" are the evolutionarily conserved group VII ethylene response factor (ERF-VII) transcription factors (TFs), which have been extensively investigated in rice and Arabidopsis. One study found that the expression of SNORKEL1 and 2 (SK1/2) was induced by the accumulation of ethylene due to submergence in deep water rice. SK $1 / 2$ activated the expression of the GA synthesis gene GA-20 oxidase (GA20ox), which promotes internode elongation and helps plants evade hypoxia stress (Hattori et al., 2009; Ayano et al., 2014). Another ERF-VII member from lowland rice, Submergence1 (Sub1A), was found to exert the antithetical function of SK1/2, which maintains "quiescence" during flooding (Xu et al., 2006; Fukao \& Bailey-Serres 2008; Niroula et al., 2012). By enhancing the mRNA levels of Slender Rice-1 (SLR1)/SLR1 Like-1 (SLRL1) and GA catabolic gene GA2ox7, Sub1A was found to inhibit GA signaling and production. Sub1A can also restrict the rate and extent of catabolism of starch and soluble sugars (Fukao \& Bailey-Serres 2008; Barding et al., 2012; Niroula et al., 2012). The five group VII ethylene-responsive TFs (ERF-VIIs) of A. thaliana, including RELATED TO APETALA2.12 (RAP2.12, AtERF75), RAP2.2 (AtERF74), RAP2.3 (AtERF72), and hypoxia responsive ERFs (HRE1/AtERF73, HRE2/AtERF71), mainly participate in the transcription of anaerobic metabolism genes to construct metabolic acclimations (Licausi et al., 2010; Gibbs et al., 2011; Licausi et al., 2011). The oxygen levels under hypoxic stress conditions influence the localization and stabilities of ERF-VIIs proteins. The stability of ERF-VII proteins is modulated by the presence of molecular oxygen via the $\mathrm{N}$-end rule protein degradation pathway. (Hinz et al., 2010; Licausi et al., 2010; Papdi et al., 2015; Prabhakar \& Semenza 2015). Trihelix TF hypoxia response attenuator 1 (HRA1) directly binds RAP2.12, which plays an important role in the N-end rule pathway (Giuntoli et al., 2014). In addition to ERF-VII and Trihelix $\mathrm{TF}$, the study of angiosperm flooding response using high-throughput sequencing identified three other significantly enriched TF motifs in submergence-upregulated family genes, namely a basic helix-loop-helix (bHLH), MYB, and WRKY-type motif. This research indicated the important roles of bHLH, MYB, and WRKY in the flooding response (Reynoso et al., 2019).

Several studies have reported the regulation mechanism of flooding tolerance in species other than rice and Arabidopsis. These researches focused on The ERF-VIIs genes too(Fan et al., 2018; Pan et al., 2019; Yu et al., 2019; Luan et al., 2020). For example, the overexpression of ZmEREB180, a maize ERF-VII member in Arabidopsis and maize, has been found to enhance the survival rate of plants after long-term waterlogging stress through the enhanced formation of adventitious roots (ARs) (Yu et al., 2019). Phenotypic and physiological analyses of transgenic Arabidopsis overexpressing barley HvERF2.11 indicated that HvERF2.11 works as a positive regulator in plant waterlogging tolerance by improving antioxidant and ADH enzyme activities (Luan et al., 2020). The overexpression of AdRAP2.3 in transgenic tobacco also improved the expression levels of waterlogging marker genes, such as PDC and ADH (Pan et al., 2019). Other studies on flooding tolerance mechanisms in non-model plants are mainly carried on by RNA sequencing, such as with Chrysanthemum morifolium, maize, cucumber, Zombi pea (Vigna vexillata), and Brassica napus (Zou et al., 2013; Arora et al., 2017; Xu et al., 2017; Zhao et al., 
124 2018; Butsayawarapat et al., 2019). These transcriptomic studies have reported similar 125 differentially expressed genes (DEGs) that function as TFs, regulators, or factors in hormone 126 synthesis and signaling, ROS-scavenging, N-end turnover, anaerobic respiration, and 127 carbohydrate metabolism. A number of studies have identified the mechanisms underlying the 128 differences in waterlogging tolerance in different varieties, including Chrysanthemum

129 morifolium and Zombi pea (Zhao et al., 2018; Butsayawarapat et al., 2019).

The molecular mechanism of flooding tolerance was uncovered by studies primarily on herbaceous plants, particularly rice and Arabidopsis. With regards to the mechanisms for flooding tolerance in trees, studies using transcriptome, physiological, and metabolome analyses have identified few key regulator genes, despite the enormous economic, ecological, and social importance of forest ecosystems (Juntawong et al., 2014; Kreuzwieser \& Rennenberg 2014; Qi et al., 2014; Zhang et al., 2017; Salvatierra et al., 2020). In general, trees have been found to survive $\mathrm{O}_{2}$ deficiency stress by slowing down the anabolic processes and maintaining a steady supply (Kreuzwieser \& Rennenberg 2014; Salvatierra et al., 2020). Transcriptome analysis on "Zhongshansa," which shows a remarkable tolerance to waterlogging, showed that ROS detoxification and energy maintenance were the primary coping mechanisms of this species.

Salix, such as the arbor willow, is a tree species that provides significant environmental protection within its ecosystems and has a strong tolerance to flooding stress (Kuzovkina \& Volk, 2009). The characterization of responses of willows to partial flooding has mainly focused on physiological aspects (Li et al., 2004; Rodríguez et al., 2018), and little is known about the responses of these trees to submergence, as well as the molecular mechanisms in submergence response. Therefore, to elucidate the genetic basis of the submergence tolerance of Salix and identify its key regulators, we analyzed the transcriptomes of roots exposed to submergence from two Salix varieties with different submergence tolerance abilities and identified several hub genes in hypoxia tolerance. Based on our findings, we propose a potential mechanism by which submergence-tolerant varieties obtain high tolerance abilities upon submergence stress.

\section{Material \& Methods}

\section{Screening submergence-tolerant and -sensitive varieties in arbor willow germplasm}

One-year-old stem cuttings (length, 8-10 cm; coarse, 2-3 mm) of thirteen arbor willow varieties (“Zhuliu," "Suliu 172," "Suliu 795," "Suliu 932," "Longzhualiu," “Dongying No. 1," "J1010," “Ziganliu," “J2187," “J2087," "Bohailiu No. 1," "Yanliu No. 1," and “287”) were immersed in water to mimic submergence stress. Three submergence stress treatment repeats and a control experiment were set for each willow variety, with 15 stem cuttings per treatment. The emergence time and number of buds and roots from the stem cuttings were counted every three days to determine the tolerant and sensitive varieties. The duration of submergence stress treatment was 66 days. During the submergence stress period, varieties with more buds/roots and longer buds/roots were characterized as tolerant to submergence. Total Chlorophyll content and Malondialdehyde (MDA) contents were measured using kits according to the manufacturer's 
164

165

instructions (Solarbio, Beijing, CN).

\section{Treatment and collection of samples for RNA sequencing}

Stem cuttings (length, $8-10 \mathrm{~cm}$; coarse, $2-3 \mathrm{~mm}$ ) of submergence-tolerant and -sensitive willow varieties were cultured in Hoagland liquid medium for $20 \mathrm{~d}$ to induce the emergence of roots and buds. The liquid medium surface was $4 \mathrm{~cm}$ above the end of the stem cuttings. The stem cuttings with newly emerged roots and buds were completely immersed in water, and root samples were collected after $0,4,12,24$, and $48 \mathrm{~h}$ of submergence stress for RNA sequencing. The samples were labeled as WR (waterlogging resistance)-CK, WR-4h, WR-12h, WR-24h, WR-48h, and waterlogging sensitive Yanliu (WSYL)-CK, WSYL-4h, WSYL-12h, WSYL-24h, and WSYL$48 \mathrm{~h}$ for the five samples from the submergence-tolerant (Suliu 795) and -sensitive (Yanliu No. 1) varieties, respectively. WR-CK sample means submergence-tolerant variety roots not treated with submergence stress; WSYL-CK sample means submergence-sensitive variety roots not treated with submergence stress. The experiments were repeated in triplicate, and a total of 30 samples were collected for RNA sequencing.

\section{RNA sequencing, data processing, and gene annotation}

Total RNA from all samples was extracted and purified using the same protocol and reagent as reported by Chen et al. (2020b). cDNA library construction was performed according to the protocol described by Chen et al. (2020b). After completing library construction, Qubit2.0 and Agilent 2100 were used to detect library concentration and library insert size; Q-PCR was used to accurately measure library concentrations for library quantification. After passing library quality inspection, the library preparations were sequenced on an Illumina platform, and pairedend reads were generated. Clean data (clean reads) were obtained from the screening of raw data (raw reads). The Q20, Q30, GC-content, and sequence duplication levels of the clean data were calculated to guarantee that all downstream analyses were based on clean data with high quality. Principal component analysis (PCA) was performed to verify the reliability of the repeated experiments.

These clean reads were then mapped to the Salix matsudana reference genome sequence V1.0 (Zhang et al., 2020). TopHat2 software was used to map the reference genome (Kim et al., 2013). Using BLAST+ (v2.2.23) software (http://blast.ncbi.nlm.nih.gov/Blast.cgi) with default parameters, gene function was annotated based on the seven databases routinely used in gene annotation (Qi et al., 2014; Zhao et al., 2018).

\section{Differential expression analysis and GO and KEGG pathway enrichment analysis on differentially expressed genes}

The gene expression levels were estimated by fragments per kilobase of transcript per million fragments mapped (Jin et al., 2017). Differential expression analysis of two conditions/groups was performed using DESeq2 (Wang et al., 2009). False discovery rate was controlled through Benjamini and Hochberg's approach by adjusting the resulting P value. Genes both with an adjusted $\mathrm{P}$ value $<0.01$, and fold change value $>2$ were confirmed as DEGs. Gene Ontology 
205 (GO) enrichment analysis of DEGs was carried out by the GOseq R packages (Young et al., 206 2010). The statistical enrichment of DEGs in the KEGG pathways was analyzed using KOBAS 207 software (Mao et al., 2005).

\section{Quantitative real-time PCR}

The expression patterns of selected candidate DEGs were verified using quantitative real-time PCR to test the reliability of RNA sequencing. Ten RNA samples for RNA sequencing were reverse transcribed, and qRT-PCR was carried out, as previously reported (Zhang et al. 2020). The actin gene (SapurV1A.0655s0050.1) in Salix was used as the reference gene. The genespecific primers for the 15 selected genes are listed in Table S1. Relative expression was measured using the $2^{-\Delta \Delta \mathrm{Ct}}$ method. The expression level of "WR-CK" was selected as the reference and normalized to a relative expression of 1.0.

\section{Co-expression gene network construction of DEGs and hub gene identification} DEGs with FPKM $(\geq 0.5)$ were used to construct the co-expression gene network by $\mathrm{R}$ package weighted gene correlation network analysis (WGCNA) (Langfelder \& Horvath 2008). The minimum module size and the minimum height for merging modules were set to 30 and 0.2 , respectively. The graphic networks on modules were visualized using Cytoscape 3.7.0 software (Shannon et al., 2003). GO and KEGG pathway enrichment analyses were performed on DEGs in modules with high correlation coefficients and corresponding lower P-values. Genes in the modules with the highest connectivity values were identified as hub genes.

\section{Expression pattern analysis of DEGs involved in hormone and metabolism} pathways related to submergence stress response

Using BLAST, we identified the S. matsudana homolog genes of key Arabidopsis metabolism and signaling pathways in flooding response, including carbohydrate metabolism, ROS signaling pathway, anaerobic respiration, N-end rule pathway, phytohormone (ethylene, GA, and salicylic acid (SA)) synthesis, and signaling pathways. DEGs from these genes were identified and their expression patterns were illustrated using the heatmap illustrator of TBtools (Chen et al., 2020a). Transcription factor prediction analysis was performed using the BMKCloud platform (www.biocloud.net). The statistical analysis of total TF genes and DEGs of TF genes was carried out based on the analysis. The expression patterns of the putative DEGs of the TFs in the RNAseq were presented by heatmaps using TBtools (Chen et al., 2020a).

\section{Results}

\section{"Suliu 795" and "Yanliu No. 1" were selected as submergence-tolerant and -} sensitive representative varieties, respectively

Thirteen varieties, namely "Zhuliu," "Suliu 172," "Suliu 795," "Suliu 932," "Longzhualiu," "Dongying No. 1," “J1010," "Ziganliu," “J2187," “J2087," "Bohailiu No. 1," "Yanliu No. 1," and "287," were selected for submergence stress experiments. 
The total root number, total root length, total shoot number, and total shoot length of 13 varieties under different submergence treatment times are summarized in Fig. 1 and Table S2. In the total root number panel, the top four varieties were "Zhuliu," "Suliu 172," "Suliu 795," and "Suliu 932" (Fig. 1a). In the total root length panel, the top four varieties were "Zhuliu," "Suliu 172," "Suliu 932," and "Suliu 795" (Fig. 1b). In the total shoot number panel, the top four varieties were "Longzhualiu," "Dongying No. 1," "Suliu 795," and "287” (Fig. 1c). In the total shoot length panel, the top four varieties were "Suliu 795," "Longzhualiu," "Zhuliu," and "Dongying No. 1" (Fig. 1d). These seven varieties had the ability to maintain root and bud growth under long-term flooding submergence. Some showed advantages in root growth ("Zhuliu," "Suliu 172," and "Suliu 932"), others advantages in bud growth ("Longzhualiu," "Dongying No. 1"), and only "Suliu 795" predominated in all four evaluation indicators. In addition to these seven varieties, another six varieties ("J1010," "Yanliu No. 1," "J2187," “J2087," "Ziganliu," and "Bohailiu No. 1") stopped growing or maintained a slow growth rate under long-term submergence. We also made analysis on two physiological indicators including total Chlorophyll content and MDA content. From the results of MDA content, we found that the MDA content in 'Suliu 795' is the lowest in all samples detected (Fig. S1a). After submergence stress, the total chlorophyll content in all samples decreased, but the smallest reduction range was found in the 'Suliu 795'. (Fig. S1b). Since "Yanliu No. 1" is a variety of Salix found in salty land, we selected "Suliu 795" and "Yanliu No. 1" as representatives for submergence-tolerant and -sensitive varieties, respectively. The growth phenotype under normal conditions (hydroponics culture) and submergence stress of "Suliu 795" and "Yanliu No. 1" are presented in Fig. 1e and Fig. 1f.

\section{RNA sequencing and data quality control}

To identify the differences in the mechanisms of the response to submergence between the sensitive and tolerant cultivars, thirty libraries (three repeat libraries for one sample) from the root tissues of the varieties, i.e., WR-CK ("Suliu 795" control), WR-4h, WR-12h, WR-24h, WR48h, WSYL-CK ("Yanliu No. 1 " control), WSYL-4h, WSYL-12h, WSYL-24h, and WSYL-48h, were constructed in this study. After controlling for the quality of the sequencing data, 192.46 $\mathrm{Gb}$ clean data were obtained using Illumina HiSeq. The minimum and average GC contents were $91.55 \%$ and $44.23 \%$, respectively (Table S3). From the alignment results using the S. matsudana genome as the reference genome, the alignment ratio of samples ranged from $71.52 \%$ to $85.99 \%$, of which approximately $90 \%$ reads were mapped to the exon region (Table S3).

An assessment of the relationships between biological replicates is essential for analyzing transcriptome sequencing data for a project with biological replicates. Thus, PCA was performed to confirm the uniformity between biological replicates of the 10 group samples (Fig. 2a). Three biological replicates from 10 groups were clustered tightly and separated distinctly from each other, indicating the reliability of our RNA-seq results.

\section{Comparative transcriptome analyses and GO annotation and pathway enrichment analysis on DEGs}


286 To identify the differences in the expression profiles between the two varieties and the dynamic 287 changes following the extension of submergence time, 25 comparisons were made to identify 288 DEGs between different treatment time points in the same variety and between the two varieties 289 (Table S4-S8), 22790 DEGs were identified from 25 comparisons (Table S9), the sequences of 290 all DEGs were listed in supplemental file S1 and S2. The 25 comparisons fell into five categories: 291 category A included 4 comparisons between different treatment time points and the control in the and WR-CK/WR-48h); category B included 4 comparisons between different treatment time points and the control in the submergence-sensitive variety "Yanliu No. 1" (WSYL-CK/WSYL4h, WSYL-CK/WSYL-12h, WSYL-CK/WSYL-24h, and WSYL-CK/WSYL-48h); category C included 5 comparisons between two varieties at 5 treatment time points (WR-CK/WSYL-CK, WR-4h/WSYL-4h，WR-12h/WSYL-12h，WR-24h/WSYL-24h， and WR-48h/WSYL-48h); category D (WR-4h/WR-12h，WR-4h/WR-24h，WR-4h/WR-48h，WR-12h/WR-24h，WR12h/WR-48h, and WR-24h/WR-48h) and category E (WSYL-4h/WSYL-12h, WSYL-4h/WSYL24h, WSYL-4h/WSYL-48h, WSYL-12h/WSYL-24h, WSYL-12h/WSYL-48h, and WSYL24h/WSYL-48h) represent comparisons between different treatment time points in "Suliu 795" and "Yanliu No. 1", respectively. The upregulated and downregulated DEGs in each comparison are shown in Fig. 2b. In category A and B, more upregulated DEGs were only found in two comparisons between the $\mathrm{CK}$ and $4 \mathrm{~h}$ treatments, while in the other six comparisons, the number of downregulated DEGs was higher than that of upregulated DEGs. These data indicate that upon longer submergence, the plant adapted to low oxygen levels by slowing down cellular activity. Except for the comparisons between the CK and $4 \mathrm{~h}$ treatments, the tolerant variety "Suliu 795" had more DEGs than the sensitive variety "Yanliu No. 1" during longer submergence stages (Fig. 2b, Table S4-S8), demonstrating that "Suliu 795" had a stronger response to submergence than "Yanliu No. 1" at the transcription level. The highest number of DEGs was observed in the WR-CK/WR-24h comparison, with 3383 upregulated DEGs and 5941 downregulated DEGs. In five comparisons in category C, an average of 5000 DEGs were found, and the WR-CK/WSYL-CK comparison had 4820 DEGs, which suggested that there were significant differences in gene expression between the two cultivars during hydroponic rooting and response to submergence stress. In categories D and $\mathrm{E}$, the smaller number of DEGs in comparisons WR-24h/WR-48h (a total of 1259 DEGs, including 747 up- and 512 downregulated genes) and WSYL-24h/WSYL-48h (a total of 490 DEGs including 220 up- and 270 downregulated genes) indicated a similar expression pattern at the $24 \mathrm{~h}$ and $48 \mathrm{~h}$ treatment time points in both varieties.

Venn diagrams were drawn to reveal overlapping DEGs in the different categories. In category A, there were 1414 overlapped DEGs, with more downregulated DEGs (800) than upregulated DEGs, whereas in sensitive variety category B the number of overlapped DEGs was 2180 , and that of the upregulated DEGs was more than twice as many as that of the downregulated DEGs (Fig. 2c). The percentages of overlapping DEGs in categories A and B were $5.19 \%$ and $9.16 \%$, respectively. In five comparisons of category $\mathrm{C}$, there were a total of 1259 overlapped DEGs (650 upregulated DEGs, 609 downregulated DEGs), accounting for only 
$3275 \%$ of the total DEGs (Fig. 2c). The GO annotations of all overlapped DEGs were listed in six 328 sheets of Table S10. From the table, we find that the top terms in biological processes (BP) and 329 molecular functions (MF) were same in all overlapped DEGs groups, which are metabolic 330 process (BP, GO:0008152) and binding (MF, GO:0003824 ).

331 To identify the major functional categories represented by the DEGs in different comparisons, 332 gene ontology (GO) enrichment analysis, including the analysis of biological processes (BP), 333 molecular functions (MF), and cellular components (CC), was performed. The results of BP 334 enrichment terms (top 20 according to P-value) from upregulated DEGs and downregulated 335 DEGs in categories $\mathrm{A}$ and $\mathrm{B}$ are illustrated in Fig. 3 and Table S11-S12. The BP term 336 "oxidation-reduction process (GO:0055114)" was found in all columns, indicating radical 337 changes in the regulation of energy metabolism under submergence stress (Fig. 3). In the tolerant 338 variety, after $4 \mathrm{~h}$ submergence treatment, the terms "negative regulation of catalytic activity 339 (GO:0043086)," "negative regulation of endopeptidase activity (GO:0010951)," "negative 340 regulation of nucleic acid-template transcription (GO:1903507)," and "regulation of protein 341 serine/threonine phosphatase activity (GO:0080163)" were enriched in upregulated DEGs, 342 indicating that plants slow down cellular activity upon stress (Fig. 3). The term "secondary 343 metabolite biosynthetic process (GO:0044550)" was enriched in both upregulated DEGs and 344 downregulated DEGs in WR-CK/WR-4h comparison, while after $8 \mathrm{~h}$ under stress conditions, 345 this term was only listed in downregulated DEGs (Fig. 3). After $12 \mathrm{~h}$ of stress, new terms 346 including "carbohydrate metabolic process (GO:0005975)," "nucleoside metabolic process 347 (GO:0009116)," "response to nitrate (GO:0010167)," "nitrate transport (GO:0015706)," and 348 "starch metabolic process (GO:0005982)" were enriched in upregulated DEGs. In comparison, in 349 WR-CK/WR-24h and WR-CK/WR-48h the term "anaerobic respiration (GO:0009061)" was 350 enriched in upregulated DEGs; the term "detection of hypoxia (GO:0070483)" was also 351 overrepresented in upregulated DEGs of WR-CK/WR-48h comparison (Fig. 3). Some 352 photosynthesis-related terms were found to be enriched in the columns from the upregulated 353 regulated DEGs of category $\mathrm{A}$. The term "photosynthesis, light harvesting in photosystem I 354 (GO:0009768)" was enriched in three comparisons, including WR-CK/WR-12h, WR-CK/WR355 24h, and WR-CK/WR-48h. Three terms, including "photosynthesis, light harvesting in 356 photosystem I (GO:0009768)," "photosynthesis (GO:0015979)," and "chlorophyll biosynthetic 357 process (GO:0015995)," were enriched in upregulated DEGs of WR-CK/WR-48h comparison 358 (Fig. 3). In the submergence-sensitive variety, several terms were identical, such as "anaerobic 359 respiration (GO:0009061), " while others were different. Photosynthesis-related terms were only 360 found in the downregulated DEGs of WYSL-CK/WYSL-4h and WYSL-CK/WYSL-12h 361 comparisons. The term "regulation of transcription, DNA-templated (GO:0006355)" was not 362 listed in category A, but was enriched in upregulated DEGs in all comparisons from category B. 363 The terms "hydrogen peroxide catabolic process (GO:0042744)" and "cellular oxidant 364 detoxification (GO:0098869)" were enriched in the upregulated DEGs of WYSL-CK/WYSL$36548 \mathrm{~h}$ but not in those of WR-CK/WR-48h (Fig. 3).

367 Large number of transcription factors is involved in the submergence stress 


\section{response in Salix}

Using BLAST, 4584 TF genes were identified from 62400 annotated genes, 1844 TF genes were DEGs, and the top five TF family categories were AP2/ERF, MYB, NAC, WRKY, and bHLH (Fig. 4a). Depending on the ratio of number of DEG TFs to that of total TFs in one TF family, the top five TF family categories were WRKY, AP2/ERF, bZIP, NAC, and MYB (Fig. 4a). The expression patterns of some TF DEGs from seven families are presented in a heatmap (Fig. 4b). The expression patterns of most TFs can be classified into three types. The first one is that gene expression was induced after a short-term submergence ( $4 \mathrm{~h}, 12 \mathrm{~h})$, but inhibited after a longterm submergence ( $24 \mathrm{~h}, 48 \mathrm{~h}$ ), which comprised the major proportion of TF DEGs. The second is that after induction by short-term submergence, the high expression level persisted throughout the whole treatment period (e.g., EVM0007332 and EVM0050990). The third is that gene expression levels were higher before stress treatment, decreased after short-term submergence, and restored after long-term submergence (e.g., EVM0010523 and EVM0046641). Some TFs, such as EVM0047172 and EVM0000045 share similar expression patterns in submergencetolerant and -sensitive varieties, in which the expression levels were also the same. Other TFs shared similar expression patterns, but the expression levels were higher in the submergencesensitive variety than that in the submergence-tolerant variety. Most TFs are members of the AP2/ERF family, such as EVM0043629, EVM0008900, and EVM0041629. A small portion of DEGs had different expression patterns in the submergence-tolerant and -sensitive varieties (e.g.,

EVM0056506, EVM0029583, and EVM0049452). Fourteen members of the AP2/ERF GroupVII were identified from the Salix genome; their expression patterns are illustrated in Fig. 4c. According to their expression patterns, 14 genes can be classified into three categories. The expression levels of four genes on the top of the heatmap changed after submergence stress, with plants subjected to $24 \mathrm{~h}$ and $48 \mathrm{~h}$ treatment showing the highest expression levels. The second category, which included six genes, had higher expression levels at all treatment time points, with little variation between the treatments. The expression levels of the other four genes were very low (Fig. 4c).

Identification on DEGs encoding for factors of carbohydrate metabolism, ROS signaling pathway, anaerobic respiration, and $\mathrm{N}$-end rule pathway

Upon submergence stress, plants regulated the carbohydrate metabolism and ROS signaling pathway to cope with the hypoxia condition via the $\mathrm{N}$-end rule pathway. We detected DEGs from these pathways, as illustrated in Fig. 5a, b. The $A D H$ gene is a marker of fermentation; five Salix $A D H$ genes with differential expression patterns were identified. Their expression levels reached a peak after $24 \mathrm{~h}$ treatment and were maintained at a higher level at $48 \mathrm{~h}$, with the exception of EVM0046399_AtADH, whose expression level at $48 \mathrm{~h}$ decreased compared to the WYSL sample. It is worth noting that the expression levels of all five members were lower after $48 \mathrm{~h}$ in the WR samples than in the WYSL samples. SUS cleaves sucrose and provides monosaccharide substrates for glycolysis under submergence. Four SUS DEGs were found, and their expression was upregulated under longer submergence stress ( $24 \mathrm{~h}$ and $48 \mathrm{~h}$ ). The 
408

expression levels of two members (EVM0011515_AtSUS1 and EVM0020822_AtSUS1) were higher in the WR samples than in the WYSL samples. Another three kinds of enzymes related to glycolysis were LDH, PDC, and AlaAT: 1 LDH, 4 PDC, and 4 AlaAT DEGs were found to be important factors in the fermentation pathway (Fig. 4a). Their expression levels were upregulated under hypoxia condition in both the WR and WYSL samples, with slight differences observed in several DEGs between WR and WRYL (e.g., EVM0013254_AlaAT and EVM0049446_AtPDC1).

DEGs coding for enzymes involved in ROX production, signaling, and ROS detoxification are shown in a heatmap (Fig. 5b). In six AOX1A members, four members had a higher expression level after $4 \mathrm{~h}$ of treatment in the WRYL samples than in the WR samples. Six AOX1A members reached the maximum expression level after $4 \mathrm{~h}$ in the WRYL samples; however, this tendency was only observed for EVM0034773_AOX1A in the WR samples. While all five RBOHD DEGs had higher levels of expression in the WR samples, there were no evident differences in the levels of three kinds of antioxidant enzymes.

The expression profiles of PCO DEGs were inhibited after short-term submergence, and then upregulated under hypoxic conditions. DEG EVM0025544_PCO1 was found to have a higher level of expression in the WSYL sample at $24 \mathrm{~h}$ and $48 \mathrm{~h}$ (Fig. 5b).

\section{Identification on DEGs encoding proteins in ethylene, GA, and salicylic acid (SA) synthesis and signaling}

Five key genes in GA synthesis, GA20OXs, were identified. The expression levels of four of the five DEGs in WSYL were found to be higher than those in the WR samples. The GA receptor DEG EVM0004859_GID1C and GA-regulated protein GASA DEG were substantially upregulated in WSYL (Fig. 5c). For ethylene synthesis, five $A C O$ genes were detected, and all five genes were found to be significantly upregulated at $4 \mathrm{~h}$. Two genes, EVM0049609_ACO and EVM0029609_ACO, were upregulated in the WR samples, with the exception of EVM0047872_ACO. For SA signaling, SAR deficient 1 (SARD1) is a key regulator of ICS1 (isochorismate synthase 1) induction and SA synthesis. In the WR-CK sample, the expression levels of SARD1 were much higher than those in WSYL-CK. After $4 \mathrm{~h}$ of submergence, three genes were downregulated in WR but upregulated in WSYL. The ICS1 gene also showed an upregulated expression pattern in WR samples at 0,4 , and $12 \mathrm{~h}$ (Fig. 5c).

\section{Hub genes were identified by co-expression network construction using WGCNA}

A co-expression network using WGCNA was constructed to investigate the interrelationships among submergence responsive genes and identify the key regulators. According to the expression patterns of 10097 DEGs (FPKM Value $>0.5$ ), a hierarchical clustering tree (dynamic hybrid tree cut algorithm) was constructed and the DEGs were divided into 16 co-expression modules, with the highest number of DEGs (2371) in the black module and the lowest number of DEGs (30) in the lightsteelbluel module (Fig. S2, Table S13). The module trait correlation heatmap was drawn to show the correlation significance between the modules and submergence stress and willow samples with different hypoxia tolerance (Fig. S3). The modules with higher 
449 correlation significance $(>0.7)$ and lower P-value $(<0.05)$, the red and green modules, 450 respectively, were selected for further analysis (Fig. S3). While the genes from red modules were 451 induced rapidly after short-term submergence, higher expression levels did not last and reduced 452 to a lower level during the rest of the submergence period. Another significant feature is that the 453 expression levels of these genes were higher in the WYSL samples than in WR samples after $4 \mathrm{~h}$ 454 submergence treatment (Fig. S4a). Genes from the green module also had significantly different 455 expression profiles in the WYSL and WR samples. The peak of the expression level of these 456 genes appeared in the WR samples without submergence stress, and then their expression levels 457 decreased following submergence stress (Fig. S4b).

458 We performed functional enrichment analysis of genes in modules. The Clusters of 459 Orthologous Groups (COG) function classification of green and red modules are presented in Fig. 460 S5 and Table S14. The top three functional classes from the green module were G (carbohydrate 461 transport and metabolism), T (signal transduction mechanisms), and Q (secondary metabolite 462 biosynthesis, transport, and catabolism), while the top three classes from the red module were G, $463 \mathrm{O}$ (post-translational modification, protein turnover, chaperones), and $\mathrm{T}$. Apart from the difference in top classes, the red and green modules had a similar enrichment pattern.

In the red and green modules, according to the $\mathrm{kME}$ value and correlation significance (Table S15), the hub genes were identified and the networks were constructed and visualized using Cytoscape 3.6.1. Eleven and five TFs were identified as hub genes for the red and green modules, respectively. In the red module, the 11 TFs included one GIF (EVM0054186), two trihelix TFs (EVM0004070, EVM0046362), three NAC TFs (EVM0056498, EVM0045598, EVM0025876), three WRKY TFs (EVM0008101, EVM0015670, EVM0006564), and two AP2/ERFs (EVM0004610, EVM0047172), among which GIF (EVM0054186), trihelix EVM0046362, and NAC EVM0056498 were located at the center of the network (Fig. 6a). In the green module, three MYBs and two NACs were illustrated at the center of the diagram (Fig. $6 \mathrm{~b})$. These TFs could be defined as candidate genes involved in the submergence stress response, and further functional evaluation of these genes will be carried out in the future.

\section{Verification of RNA sequencing results by qRT-PCR analysis of key genes}

We selected 15 genes from the total number of DEGs identified for qRT-PCR to verify the reliability of RNA sequencing. The names and annotations of 15 genes, including putative hub TF genes, important factors in fermentation, hormone synthesis and signaling pathways, and oxygen level sensing are listed in Table S16. The expression patterns of these 15 genes are shown in Figure 7. The qRT-PCR results showed that the expression profiles of all genes and the changes in expression after different periods of stress treatment were consistent with FPKM values (Table S9).

\section{Discussion}

\section{Submergence-tolerant varieties are capable of continuous growth under long-term hypoxia}


489 According to a previous study, willows have one of the highest tolerances to flooding stress in 490 trees (Mozo et al., 2021). However, the growth characteristics of willows during long-term 491 submergence have not been reported previously. In our submergence experiments, different Salix 492 varieties were found to have distinct growth capacities under long-term submergence stress. 493 Submergence-tolerant varieties, such as "Suliu 795," were able to maintain growth even after 494 submergence lasting two months. When comparing the shoot length between $60 \mathrm{~d}$ and $66 \mathrm{~d}$, the 495 length of the shoots increased by ten percent, suggesting that "Suliu 795" could cope with 496 hypoxic conditions (Fig. 1). Comparative transcriptomic analyses of submergence-tolerant and sensitive varieties will help us uncover the mechanisms underlying these responses.

\section{RNA sequencing results were reliable and indicated that large number of DEGs was involved in the submergence stress response}

Two varieties with contrasting submergence tolerance abilities were used to perform a comparative transcriptome analysis. Five samples were collected at the $0,4,8,24$, and $48 \mathrm{~h}$ submergence time points from one variety, and a total of ten samples were collected, where every sample had three replicates. The clean data from RNA sequencing had a high Q30 value and a high alignment ratio to the reference genome. PCA analysis showed that the three repeats from one sample clustered tightly together and in different samples separately (Fig. 2a). Verification of the expression of 15 genes by qRT-PCR showed that almost all genes had a similar expression oscillation pattern with the FPKM value obtained by high-throughput sequencing during the long-term submergence stress (Fig. 7). The results of these analyses indicated that the RNA sequencing data were reliable and could be used to conduct a thorough investigation of the molecular mechanisms of willow submergence. A large number of DEGs were identified between different time points after submergence stress in one variety (categories A, B, D, E) and also between the same time points from two varieties (category C) (Fig. 2b). The number of DEGs peaked after submergence for $24 \mathrm{~h}$ in both varieties, while the number of DEGs from two comparisons between $24 \mathrm{~h}$ and $48 \mathrm{~h}$ in the two varieties was the smallest. These data demonstrate that the regulation of gene expression peaked at $24 \mathrm{~h}$ in response to submergence stress, after which plants maintained their status in response to hypoxia. As a result, few DEGs were found in comparison between $24 \mathrm{~h}$ and $48 \mathrm{~h}$ (Fig. 2b). Shortly after submergence, the DEGs included more upregulated genes than downregulated genes. By contrast, after $12 \mathrm{~h}$ of submergence, the number of upregulated genes decreased and the number of downregulated genes increased. As a result, the DEGs included more downregulated genes than upregulated genes. These data indicate that after short-term submergence, plants began to attenuate the biological activities to adapt to the long-term submergence stress by downregulating the expression of some genes.

The Venn diagram signified the common and unique DEGs of the different comparisons (Fig. 2c). The Venn diagrams based on the comparisons between categories A, B, and C show that the portions of common DEGs for four or five comparisons were very small, with less than $10 \%$ of the total DEGs (Fig. 2c). The lower percentage of overlapped DEGs in categories A and B (5.19\% and $9.16 \%$, respectively) indicated that the gene expression changed markedly at 
530 different time points under submergence stress. Tolerant varieties mobilized more dynamic gene 531 expression changes in response to submergence stress by decreasing the gene expression levels. 532 The 5\% common DEGs in category $\mathrm{C}$ implied that there were contrasting gene expression 533 patterns as a result of the different willow varieties and different submergence treatment times 534 (Fig. 2c).

535

GO analysis of DEGs revealed the molecular mechanisms underlying the submergence stress response

538 After submergence, the BP term "oxidation-reduction process (GO:0055114)" was enriched in DEGs of all comparisons from categories $\mathrm{A}$ and $\mathrm{B}$, indicating that from the beginning and through the $48 \mathrm{~h}$ submergence, the status of energy metabolism was always in dynamic change under submergence stress. At the beginning of the stress $(4 \mathrm{~h})$, the tolerant variety "Suliu 795 " quickly initiated the braking action on the cellular activity through the enrichment of three GO terms containing "negative regulation (GO:0043086, GO:0010951,GO:1903507)." Thereafter, plants adjust their metabolic strategy by increasing the expression of genes from the categories "carbohydrate metabolic process (GO:0005975)," "nucleoside metabolic process (GO:0009116)," "response to nitrate (GO:0010167)," "nitrate transport (GO:0015706)," and "starch metabolic process (GO:0005982),"suggesting that plants begin the $\mathrm{C}$ and $\mathrm{N}$ destructive metabolism to provide the raw material for the anaerobic respiration under longer-term submergence stress. After submergence for $24 \mathrm{~h}$, the term "anaerobic respiration (GO:0009061)" was enriched in the upregulated DEGs of WR-CK/WR-24h, indicating that tolerant plants convert aerobic metabolism to anaerobic metabolism successfully under hypoxia stress. To adapt to a long-term hypoxic environment, plants survive by generating oxygen through photosynthesis, with photosynthesis-related terms being found to be enriched in DEGs from WR-CK/WR-48h. From the enriched GO terms of DEGs from categories A and B, some identical terms (labeled blue) were added in the corresponding comparison from categories A and B, suggesting both varieties had a similar mechanism in the response to submergence stress (Fig. 3). Significant differences in enriched terms were also revealed (labeled red) (Fig. 3). The expression of the term "regulation of transcription, DNA-templated (GO:0006355)," which was enriched in the upregulated DEGs of all comparisons from category B, was found to be increased in many TFs after stress. The terms "hydrogen peroxide catabolic process (GO:0042744)" and "cellular oxidant detoxification (GO:0098869)" enriched in the upregulated DEGs of WYSL-CK/WYSL$48 \mathrm{~h}$ also showed that sensitive plants did not establish the metabolic balance and energy saving mechanism after $48 \mathrm{~h}$ of submergence.

\section{TFs played important roles in the submergence stress response}

566 A previous study found that four classes of TFs, including AP2/ERF, bHLH, WRKY, and MYB, 567 played significant roles in the flooding response circuitry of angiosperms (Reynoso et al., 2019). 568 In the present study, 40\% (1844 out of 4584) of annotated TF genes were DEGs, consistent with 569 previously reported results. AP2/ERF, bHLH, WRKY, and MYB were the four top TF family 570 categories. Differential expression patterns of DEG TFs implied their roles in routine regulation 
571 patterns in response to submergence stress, as well as in differential regulation in different

572 varieties, resulting in differences in the tolerance ability of plants. The roles of ERFVII members

573 in response to waterlogging stress have been extensively studied in rice and Arabidopsis (Fukao 574 \& Bailey-Serres 2008; Barding et al., 2012; Niroula et al., 2012; Gibbs et al., 2011; Licausi et al.,

575 2011). The expression patterns of 14 SmERFVII were detected, and only four members had 576 upregulated expression profiles under submergence stress, as well as a higher FPKM value. 577 However, similar expression patterns in WR and WYSL willows suggest that they were not the 578 key regulators in increasing tolerance to submergence stress in submergence-tolerant willows. 579 Several hub TF genes were identified after WGCNA analysis, with hub TFs from green and red 580 modules mostly belonging to the MYB, AP2/ERF, WRKY, NAC, and Trihelix TF families. 581 These TF genes possessed different expression profiles in the WR and WYSL willows, which 582 may provide a molecular basis for differences in the tolerance of WR and WYSL. Although 583 Trihelix TF, such as hypoxia response attenuator 1 (HRA1) does not belong to the five top TF 584 family members, it is also a crucial component in hypoxia status sensing and cell activity 585 regulation network. Further exploration of these TFs will uncover the mechanisms underlying 586 the differences in the tolerance activities of different willows.

\section{Submergence-tolerant willow "Suliu 795" may gain a higher tolerance via unique mechanisms}

590 From the annotation of DEGs and the specific expression patterns of different modules, we found that WR plants may gain a higher submergence tolerance through several mechanisms (Fig. 8). First, the expression profiles of genes from the green and red modules were specific to WR and WYSL, respectively. In the green module, the group of genes was more highly expressed in the WR-CK sample; in the red module, the other group of genes was only substantially upregulated in WYSL-4h. The two group genes shared two COG function classification terms, "carbohydrate transport and metabolism" and "signal transduction mechanisms." This implies that during the hydroponics culture stage in WR, hydroponics may mimic waterlogging stress. WR plants were very sensitive to low oxygen stress and initiated the appropriate mechanisms in advance to prepare for subsequent submergence stress.

Second, under longer submergence stress, the fermentation pathway was important for plants to survive, and the expression level of key genes in the fermentation pathway was indispensable. Five ADH genes, marker genes of fermentation, had a lower expression level at $48 \mathrm{~h}$ in the WR samples than in the WYSL samples, especially EVM0046399, whose expression declined sharply at $48 \mathrm{~h}$ compared with $24 \mathrm{~h}$. Similar changes in gene expression were observed in the EVM0013254_AlaAT and EVM0049446_AtPDC1 genes. In some hypoxia-tolerant genotypes, the genes from the glycolysis pathway must be downregulated after long-term stress to maintain a continuous glycolytic flux to ensure the energy pool needed for the processes expression in WR plants is required to maintain energy homeostasis, which is essential to avoid 
610 the detrimental effects of energy depletion under hypoxic stress (Salvatierra et al., 2020).

611 Third, the genes responsible for ethylene, GA, and SA signaling were also found to have 612 different expression patterns in the WR and WYSL plants. Ethylene acts as the primary signal 613 for the majority of adaptations to flooding (Loreti et al., 2016). The ethylene synthesis genes, 614 EVM0047872_ACO and EVM0029609_ACO, increased in expression earlier in WR, at the $0 \mathrm{~h}$ 615 and $4 \mathrm{~h}$ time points. By contrast, GA synthesis and signaling were enhanced in WYSL but 616 reduced in WR, while SA and ethylene hormone synthesis were increased in WR. Willow plants

617 may carry out the "quiescence" (low- $\mathrm{O}_{2}$ quiescence syndrome, LOQS) strategy, by responding 618 quickly to submergence, with WR plants synthesizing ethylene quickly and inhibiting the GA 619 product to suppress growth and maintain energy homeostasis to survive under conditions of 620 long-term submergence. In addition to saving energy, WR plants may also create new sources of 621 energy by obtaining oxygen via enhancing the expression of genes associated with 622 photosynthesis and establishing the photosynthesis system in their roots.

\section{Conclusion}

625 In this study, the expression of a large number of genes was found to undergo significant 626 changes under conditions of flooding stress, with a small proportion of shared genes between 627 submergence-tolerant and -sensitive varieties. Genes were found to be enriched in several 628 categories of GO terms, including "oxidation-reduction process (GO:0055114)," "secondary 629 metabolite biosynthetic process (GO:0044550)," "regulation of transcription, DNA-templated 630 (GO:0006355)", and "anaerobic respiration (GO:0009061) ". A total of 1844 TF genes were 631 identified as DEGs, with most belonging to the TF families of WRKY, AP2/ERF, bZIP, NAC, 632 and MYB. Three different types of expression profiles of TFs provided a basis for the molecular 633 mechanisms underlying the regulation of responses to flooding stress in willows, highlighting the 634 different submergence tolerance abilities for the different varieties. WGCNA analysis revealed 635 that several members of these families acted as hub genes in the regulation of the response to 636 submergence stress. In addition to the differential expression pattern of TFs in the submergence637 tolerant and -sensitive varieties, the submergence-tolerant varieties may use three additional 638 strategies to exert higher submergence tolerant activities. The first one is the hypersensitivity to 639 low oxygen levels and a quick response to submergence stress; the second one is a steady supply 640 of carbohydrates by slowing down the anabolic processes under hypoxic stress; the third one is 641 keeping "quiescence" through fine-tuning the equilibrium between phytohormones including GA, 642 SA and ethylene. Fourthermore, these plants create new sources of energy and obtain oxygen by 643 enhancing the photosynthesis system gene expression and establishing a photosynthesis system 644 in their roots (Fig.8). Moreover, further functional analyses on candidate hub genes we 645 identified in this study will help to reveal the mechanism of submergence tolerance regulation in 646 Salix matsudana.

648 Acknowledgements We thank Liwen Bianji, Edanz Editing China for editing the English text 649 of a draft of this manuscript. 
650

651

652

653

654

655

656

657

658

659

660

661

662

663

664

665

666

667

668

669

670

671

672

673

674

675

676

677

678

679

680

681

682

683

684

685

686

687

688

689

\section{ADDITIONAL INFORMATION AND DECLARATIONS}

\section{Funding}

This work is funded by the earmarked fund for the National Natural Science Foundation of China (grant no. 31971681), and by Technology Innovation Center for Land Spatial Ecorestoration in Metropolitan Area, Ministry of Natural Resources and the Fundamental Research Funds for the Central Universities (grant no. CXZX2021A03), and by the Basic Science Research Project of Nantong City (grant no. JC2020157) and by the Jiangsu Provincial Key Projects of Students Innovation and Entrepreneurship Training Program (202110304020Z). The funders had no role in study design, data collection and analysis, decision to publish, or preparation of the manuscript.

\section{Competing Interests}

The authors declare there are no competing interests.

\section{Data Availability}

The S. matsudana genome sequences are available at NCBI: PRJNA687297;

The Raw RNA sequencing data is available at the CNGB Sequence Archive (CNSA) of China National GeneBank DataBase (CNGBdb): CNP0002062;

The Assembled Sequences of Transcriptome (TSA) is available at the CNGB Sequence Archive (CNSA) of China National GeneBank DataBase (CNGBdb): CNA0038268.

\section{References}

Akman M, Bhikharie AV, McLean EH, Boonman A, Visser EJ, Schranz ME, van Tienderen PH.. (2012) Wait or escape? Contrasting submergence tolerance strategies of Rorippa amphibia, Rorippa sylvestris and their hybrid. Ann Bot. 109: 1263-1276 https:// doi:10.1093/aob/mcs059.

Arora K, Panda KK, Mittal S, Mallikarjuna MG, Rao AR, Dash PK, Thirunavukkarasu N. (2017) RNAseq revealed the important gene pathways controlling adaptive mechanisms under waterlogged stress in maize. Sci Rep. 7: 10950 https://doi/10.1038/s41598-017-10561-1.

Ayano M, Kani T, Kojima M, Sakakibara H, Kitaoka T, Kuroha T, Angeles-Shim RB, Kitano H, Nagai K, Ashikari M. (2014) Gibberellin biosynthesis and signal transduction is essential for internode elongation in deepwater rice. Plant Cell Environ 37: 2313-2324 https://doi/10.1111/pce.12377.

Barding GA, Jr., Fukao T, Béni S, Bailey-Serres J, Larive CK. (2012) Differential metabolic regulation governed by the rice SUB1A gene during submergence stress and identification of alanylglycine by 1H NMR spectroscopy. J Proteome Res. 11: 320-330 https://doi/10.1021/pr200919b.

Butsayawarapat P, Juntawong P, Khamsuk O, Somta P. (2019) Comparative transcriptome analysis of waterlogging-sensitive and tolerant zombi pea (Vigna Vexillata) reveals energy conservation and root plasticity controlling waterlogging tolerance. Plants (Basel, Switzerland) 8(8):264. https://doi/10.3390/plants8080264.

Calvo-Polanco M, Señorans J, Zwiazek JJ. (2012) Role of adventitious roots in water relations of tamarack (Larix laricina) seedlings exposed to flooding. BMC Plant Biol. 12: 99 https: //doi/10.1186/1471- 
690

691

692

693

694

695

696

697

698

699

700

701

702

703

704

705

706

707

708

709

710

711

712

713

714

715

716

717

718

719

720

721

722

723

724

725

726

727

728

729

730

2229-12-99.

Chen C, Chen H, Zhang Y, Thomas HR, Frank MH, He Y, Xia R. (2020a). TBtools: An Integrative Toolkit Developed for Interactive Analyses of Big Biological Data. Mol Plant 13: 1194-202 https://doi.org/10.1016/j.molp.2020.06.009.

Chen Y, Jiang Y, Chen Y, Feng W, Liu G, Yu C, Lian B, Zhong F, Zhang J. (2020b). Uncovering candidate genes responsive to salt stress in Salix matsudana (Koidz) by transcriptomic analysis. PLoS One 15: e0236129 https://doi/10.1371/journal.pone.0236129.

Fan W, Yang Y, Wang Z, Yin Y, Yu C, Shi Q, Guo J, Xuan L, Hua J (2018) Molecular cloning and expression analysis of three ThERFs involved in the response to waterlogging stress of Taxodium 'Zhongshanshan406', and subcellular localization of the gene products. Peer J. 6: e4434 https://doi/10.7717/peerj.4434.

Fukao T, Bailey-Serres J. (2008) Submergence tolerance conferred by Sub1A is mediated by SLR1 and SLRL1 restriction of gibberellin responses in rice. Proc Natl Acad Sci. USA 105: 16814-16819 https://doi/10.1073/pnas.0807821105.

Fukao T, Barrera-Figueroa BE, Juntawong P, Pena-Castro JM. (2019) Submergence and waterlogging stress in plants: A review highlighting research opportunities and understudied Aspects. Front Plant Sci. 10: 340 https://doi/10.3389/fpls.2019.00340.

Fukao T, Xu K, Ronald PC, Bailey-Serres J. (2006) A variable cluster of ethylene response factor-like genes regulates metabolic and developmental acclimation responses to submergence in rice. Plant Cell 18: 2021-2034 https://doi/10.1105/tpc.106.043000.

Gibbs DJ, Lee SC, Isa NM, Gramuglia S, Fukao T, Bassel GW, Correia CS, Corbineau F, Theodoulou FL, Bailey-Serres J, Holdsworth MJ. (2011) Homeostatic response to hypoxia is regulated by the Nend rule pathway in plants. Nature 479: 415-418 https:// doi/10.1038/nature10534.

Giuntoli B, Lee SC, Licausi F, Kosmacz M, Oosumi T, van Dongen JT, Bailey-Serres J, Perata P. (2014) A trihelix DNA binding protein counterbalances hypoxia-responsive transcriptional activation in Arabidopsis. PLoS Biol. 12: e1001950 https://doi/10.1371/journal.pbio.1001950.

Hattori Y, Nagai K, Furukawa S, Song XJ, Kawano R, Sakakibara H, Wu J, Matsumoto T, Yoshimura A, Kitano H, Matsuoka M, Mori H, Ashikari M. (2009) The ethylene response factors SNORKEL1 and SNORKEL2 allow rice to adapt to deep water. Nature 460: 1026-1030 https://doi/10.1038/nature08258.

Hinz M, Wilson IW, Yang J, Buerstenbinder K, Llewellyn D, Dennis ES, Sauter M, Dolferus R. (2010) Arabidopsis RAP2.2: an ethylene response transcription factor that is important for hypoxia survival. Plant Physiol. 153: 757-772 https://doi/10.1104/pp.110.155077.

Ismond KP, Dolferus R, de Pauw M, Dennis ES, Good AG. (2003) Enhanced low oxygen survival in Arabidopsis through increased metabolic flux in the fermentative pathway. Plant physiol. 132: 1292-302 https://doi/10.1104/pp.103.022244.

Jackson MB. (2008) Ethylene-promoted elongation: an adaptation to submergence stress. Ann Bot. 101: 229-248 https://doi/10.1093/aob/mcm237.

Jin H, Wan YW, Liu Z. (2017) Comprehensive evaluation of RNA-seq quantification methods for linearity. BMC Bioinformatics 18: 117 https://doi/10.1186/s12859-017-1526-y.

Juntawong P, Sirikhachornkit A, Pimjan R, Sonthirod C, Sangsrakru D, Yoocha T, Tangphatsornruang S, 
Srinives P. (2014) Elucidation of the molecular responses to waterlogging in Jatropha roots by transcriptome profiling. Front Plant Sci. 5: 658 https://doi/10.3389/fpls.2014.00658.

Kato-Noguchi H, Morokuma M. (2007) Ethanolic fermentation and anoxia tolerance in four rice cultivars. J Plant Physiol. 164: 168-73 https://doi/10.1016/j.jplph.2005.09.017.

Kim D, Pertea G, Trapnell C, Pimentel H, Kelley R, Salzberg SL. (2013) TopHat2: accurate alignment of transcriptomes in the presence of insertions, deletions and gene fusions. Genome Biol. 14: R36. https://doi/10.1186/gb-2013-14-4-r36.

Kreuzwieser J, Rennenberg H. (2014) Molecular and physiological responses of trees to waterlogging stress. Plant Cell Environ. 37: 2245-2259 https://doi/10.1111/pce.12310.

Kuzovkina, Y., and Volk, T. A. (2009). The characterization of willow (Salix L.) varieties for use in ecological engineering applications: co-ordination of structure, function and autecology. Ecol. Eng. 35, 1178-1189. doi: 10.1016/j.ecoleng.2009.03.010

Langfelder P, Horvath S. (2008) WGCNA: an R package for weighted correlation network analysis. BMC Bioinformatics 9: 559 https://doi/10.1186/1471-2105-9-559.

Li, S., Pezeshki, S. R., Goodwin, S., and Shields, F. D. (2004). Physiological responses of black willow (Salix nigra) cuttings to a range of soil moisture regimes. Photosynthetica 42, 585-590. doi: 10.1007/S11099-005-0017-y

Licausi F, Kosmacz M, Weits DA, Giuntoli B, Giorgi FM, Voesenek LA, Perata P, van Dongen JT. (2011) Oxygen sensing in plants is mediated by an $\mathrm{N}$-end rule pathway for protein destabilization. Nature 479: 419-422 https://doi/10.1038/nature10536.

Licausi F, van Dongen JT, Giuntoli B, Novi G, Santaniello A, Geigenberger P, Perata P. (2010) HRE1 and HRE2, two hypoxia-inducible ethylene response factors, affect anaerobic responses in Arabidopsis thaliana. Plant J. 62: 302-315 https://doi/10.1111/j.1365-313X.2010.04149.x.

Loreti E, van Veen H, Perata P. (2016) Plant responses to flooding stress. Curr Opin Plant Biol. 33: 64-71 https://doi/10.1016/j.pbi.2016.06.005.

Luan H, Guo B, Shen H, Pan Y, Hong Y, Lv C, Xu R. (2020) Overexpression of barley transcription factor HvERF2.11 in Arabidopsis enhances plant waterlogging tolerance. Int J Mol Sci 21 21:1982. https://doi/10.3390/ijms21061982.

Mao X, Cai T, Olyarchuk JG, Wei L. (2005) Automated genome annotation and pathway identification using the KEGG Orthology $(\mathrm{KO})$ as a controlled vocabulary. Bioinformatics 21: 3787-3793 https://doi/10.1093/bioinformatics/bti430.

Mozo I, Rodríguez ME, Monteoliva S, Luquez VMC. (2021) Floodwater depth causes different physiological responses during post-flooding in willows. Front Plant Sci. 21;12:575090. https://doi/10.3389/fpls.2021.575090.

Niroula RK, Pucciariello C, Ho VT, Novi G, Fukao T, Perata P. (2012) SUB1A-dependent and independent mechanisms are involved in the flooding tolerance of wild rice species. Plant J. 72 : 282-93 https://doi/10.1111/j.1365-313X.2012.05078.x.

Pan DL, Wang G, Wang T, Jia ZH, Guo ZR, Zhang JY. (2019) AdRAP2.3, a novel ethylene response factor VII from Actinidia deliciosa, enhances waterlogging resistance in transgenic tobacco through improving expression levels of PDC and ADH genes. Int $\mathrm{J}$ Mol Sci. 20: 1189 https://doi/10.3390/ijms20051189. 
Papdi C, Pérez-Salamó I, Joseph MP, Giuntoli B, Bögre L, Koncz C, Szabados L. (2015) The low oxygen, oxidative and osmotic stress responses synergistically act through the ethylene response factor VII genes RAP2.12, RAP2.2 and RAP2.3. Plant J. 82: 772-84 https://doi/10.1111/tpj.12848.

Prabhakar NR, Semenza GL. (2015) Oxygen sensing and homeostasis. Physiology (Bethesda, Md.) 30: 340-348 https://doi/10.1152/physiol.00022.2015.

Qi B, Yang Y, Yin Y, Xu M, Li H. (2014) De novo sequencing, assembly, and analysis of the Taxodium 'Zhongshansa' roots and shoots transcriptome in response to short-term waterlogging. BMC Plant Biol. 14: $201 \mathrm{https} / / / \mathrm{doi} / 10.1186 / \mathrm{s} 12870-014-0201-\mathrm{y}$.

Qi X, Li Q, Shen J, Qian C, Xu X, Xu Q, Chen X. (2020) Sugar enhances waterlogging-induced adventitious root formation in cucumber by promoting auxin transport and signalling. Plant Cell Environ. 43: 1545-1557 https://doi/10.1111/pce.13738.

Reynoso MA, Kajala K, Bajic M, West DA, Pauluzzi G, Yao Al, Hatch K, Zumstein K, Woodhouse M, Rodriguez-Medina J, Sinha N, Brady SM, Deal RB, Bailey-Serres J. (2019) Evolutionary flexibility in flooding response circuitry in angiosperms. Science 365: 1291-1295 https://doi/10.1126/science.aax8862.

Rich SM, Ludwig M, Colmer TD. (2012) Aquatic adventitious root development in partially and completely submerged wetland plants Cotula coronopifolia and Meionectes brownii. Ann Bot. 110: 405-414 https://doi/10.1093/aob/mcs051.

Rodríguez, M. E., Doffo, G. N., Cerrillo, T., and Luquez, V. M. C. (2018).Acclimation of cuttings of willow genotypes to flooding depth level. New For. 49, 415-427. doi: 10.1007/s11056-018-9627-7

Salvatierra A, Toro G, Mateluna P, Opazo I, Ortiz M, Pimentel P. (2020) Keep calm and survive: Adaptation strategies to energy crisis in fruit trees under root hypoxia. Plants (Basel, Switzerland) 9(9):1108 https://doi/10.3390/plants9091108.

Sasidharan R, Bailey-Serres J, Ashikari M, Atwell BJ, Colmer TD, Fagerstedt K, Fukao T, Geigenberger P, Hebelstrup KH, Hill RD, Holdsworth MJ, Ismail AM, Licausi F, Mustroph A, Nakazono M, Pedersen O, Perata P, Sauter M, Shih MC, Sorrell BK, Striker GG, van Dongen JT, Whelan J, Xiao S, Visser EJW, Voesenek LACJ. (2017) Community recommendations on terminology and procedures used in flooding and low oxygen stress research. New Phytol. 214: 1403-1407 https://doi/10.1111/nph.14519.

Sauter M. (2013) Root responses to flooding. Curr Opin Plant Biol. 16: 282-286 https://doi/10.1016/j.pbi.2013.03.013.

Shannon P, Markiel A, Ozier O, Baliga NS, Wang JT, Ramage D, Amin N, Schwikowski B, Ideker T. (2003) Cytoscape: a software environment for integrated models of biomolecular interaction networks. Genome Res. 13: 2498-2504 https://doi/10.1101/gr.1239303.

Visser EJ, Bögemann GM. (2006) Aerenchyma formation in the wetland plant Juncus effusus is independent of ethylene. New Phytol. 171: 305-314 https:// doi/10.1111/j.14698137.2006.01764.x.

Voesenek LA, Bailey-Serres J. (2015) Flood adaptive traits and processes: an overview. New Phytol. 206: 57-73 https:// doi/10.1111/nph.13209.

Wang L, Feng Z, Wang X, Wang X, Zhang X. (2009) DEGseq: an R package for identifying differentially expressed genes from RNA-seq data. Bioinformatics 26: 136-138 https:// 
813

814

815

816

817

818

819

820

821

822

823

824

825

826

827

828

829

830

831

832

833

834

835

836

837

838

839

840

841

842

843

doi/10.1093/bioinformatics/btp612.

Xu K, Xu X, Fukao T, Canlas P, Maghirang-Rodriguez R, Heuer S, Ismail AM, Bailey-Serres J, Ronald PC, Mackill DJ. (2006) Sub1A is an ethylene-response-factor-like gene that confers submergence tolerance to rice. Nature 442: 705-708 https:// doi/10.1038/nature04920.

Xu X, Chen M, Ji J, Xu Q, Qi X, Chen X. (2017) Comparative RNA-seq based transcriptome profiling of waterlogging response in cucumber hypocotyls reveals novel insights into the de novo adventitious root primordia initiation. BMC Plant Biol. 17: 129 https:// doi/10.1186/s12870-0171081-8.

Young MD, Wakefield MJ, Smyth GK, Oshlack A. (2010) Gene ontology analysis for RNA-seq: accounting for selection bias. Genome Biol. 11: R14 https://doi/10.1186/gb-2010-11-2-r14.

Yu F, Liang K, Fang T, Zhao H, Han X, Cai M, Qiu F. (2019) A group VII ethylene response factor gene, ZmEREB180, coordinates waterlogging tolerance in maize seedlings. Plant Biotechnol J. 17: 2286-2298 https:// doi/10.1111/pbi.13140.

Zhang J, Yuan H, Li Y, Chen Y, Liu G, Ye M, Yu C, Lian B, Zhong F, Jiang Y, Xu J. (2020) Genome sequencing and phylogenetic analysis of allotetraploid Salix matsudana Koidz. Hortic Res. 7: 201 https:// doi/10.1038/s41438-020-00424-8.

Zhang P, Lyu D, Jia L, He J, Qin S. (2017) Physiological and de novo transcriptome analysis of the fermentation mechanism of Cerasus sachalinensis roots in response to short-term waterlogging. BMC Genomics 18: 649 https://doi/10.1186/s12864-017-4055-1.

Zhao N, Li C, Yan Y, Cao W, Song A, Wang H, Chen S, Jiang J, Chen F. (2018) Comparative transcriptome analysis of waterlogging-sensitive and waterlogging-tolerant chrysanthemum morifolium cultivars under waterlogging stress and reoxygenation conditions. Int $\mathrm{J}$ Mol Sci 19(5):1455 https://doi/10.3390/ijms19051455.

Zhou W, Chen F, Meng Y, Chandrasekaran U, Luo X, Yang W, Shu K. (2020) Plant waterlogging/flooding stress responses: From seed germination to maturation. Plant Physiol Biochem. 148:228-236. https://doi/10.1016/j.plaphy.2020.01.020.

Zou X, Tan X, Hu C, Zeng L, Lu G, Fu G, Cheng Y, Zhang X. (2013) The transcriptome of Brassica napus L. roots under waterlogging at the seedling stage. Int J Mol Sci. 14: 2637-2651 https:// doi/10.3390/ijms14022637. 
844 Figure legends

845

Figure 1: Four growth indicators of the arbor willow germplasm under submergence stress, and the phenotypes of the submergence-tolerant and -sensitive varieties. Thirteen varieties selected for the submergence stress experiments and four indicators, including total root number, total root length, shoot number, and shoot length, are listed: (a) total root number; (b) total root length; (c) total shoot number; (d) total shoot length; (e) growth phenotype under control condition (hydroponics culture) and submergence stress of "Suliu 795" (66 days); (f) growth phenotype under control condition (hydroponics culture) and submergence stress of "Yanliu No. 1 " (66 days) (f).

Figure 2: Statistical analysis on RNA sequencing results. (a) PCA analysis. (b) Statistical analysis of differentially expressed genes (DEGs) from 25 comparisons (5 categories). (c) Venn diagrams of upregulated and downregulated DEGs from the comparisons of categories A, B, and C.

Figure 3: Biological processes in gene ontology (GO) enrichment analysis of DEGs during submergence stress in the submergence-tolerant and -sensitive varieties of Salix.

GO enrichment analysis was performed using Blast2GO. Only significantly enriched terms (top twenty) with corrected $\mathrm{P}<0.05$ were indicated. The color and size of each point represents the $-\log 10$ (FDR) values and enrichment scores. A higher $-\log 10$ (FDR) value and enrichment score indicate a greater degree of enrichment. Red and blue labeled-terms represent the different and identical terms in different comparisons, respectively.

Figure 4: Statistical analysis and heatmap illustration on TF DEGs. (a) Statistical analysis of transcription factor (TF) differentially expressed genes (DEGs). (b) Heatmap illustration on the expression profiles of some TF DEGs from seven families. (c) Heatmap illustration of the expression profiles of 14 members from the AP2/ERF GroupVII subfamily.

Figure 5: Heatmap analysis of differentially expressed genes (DEGs) encoding for important players in submergence response. (a) Heatmap analysis of differentially expressed genes (DEGs) encoding for important factors in carbohydrate metabolism and anaerobic respiration. (b) ROS signaling pathway and N-end rule pathway. (c) Ethylene, GA, and SA synthesis and signaling.

Figure 6: Identification of hub genes in co-expression network under submergence stress. (a) Eleven hub genes were identified from the red module. (b) Five hub genes were identified from the green module. The co-expression network was analyzed using WGCNA software and the graphic network was created by Cytoscape. 
884 Figure 7: Verification of the DEGs with differentially expressed patterns under 885 submergence stress by quantitative real-time PCR.

886 The gene expression profiles were evaluated using the $2^{-\Delta \Delta \mathrm{Ct}}$ method, and the control values

887 were normalized to 1 . Three biological replicates were performed for each sample. Bars 888 represent the standard deviation of the mean.

889 Figure 8: The potential mechanisms for Submergence-tolerant willow "Suliu 795" gaining 890 a higher tolerance. 


\section{Figure 1}

Figure 1: Four growth indicators of the arbor willow germplasm under submergence stress, and the phenotypes of the submergence-tolerant and -sensitive varieties

Thirteen varieties selected for the submergence stress experiments and four indicators, including total root number, total root length, shoot number, and shoot length, are listed: (a) total root number; (b) total root length; (c) total shoot number; (d) total shoot length; (e) growth phenotype under control condition (hydroponics culture) and submergence stress of "Suliu 795" (66 days); (f) growth phenotype under control condition (hydroponics culture) and submergence stress of "Yanliu No. 1" (66 days) (f). 


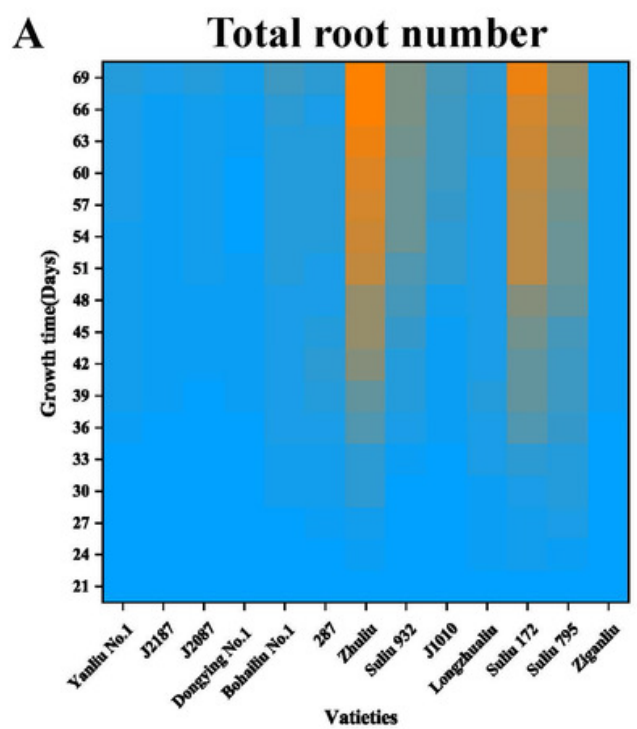

C

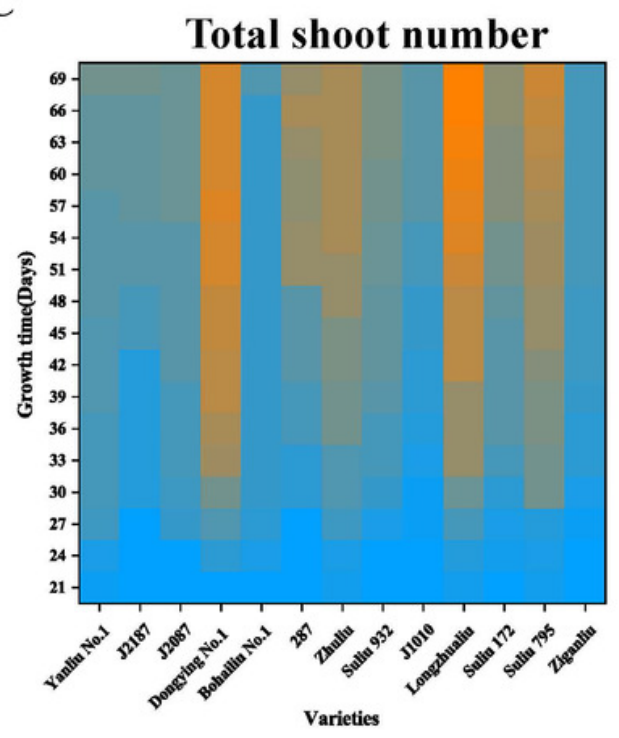

$\mathbf{E}$

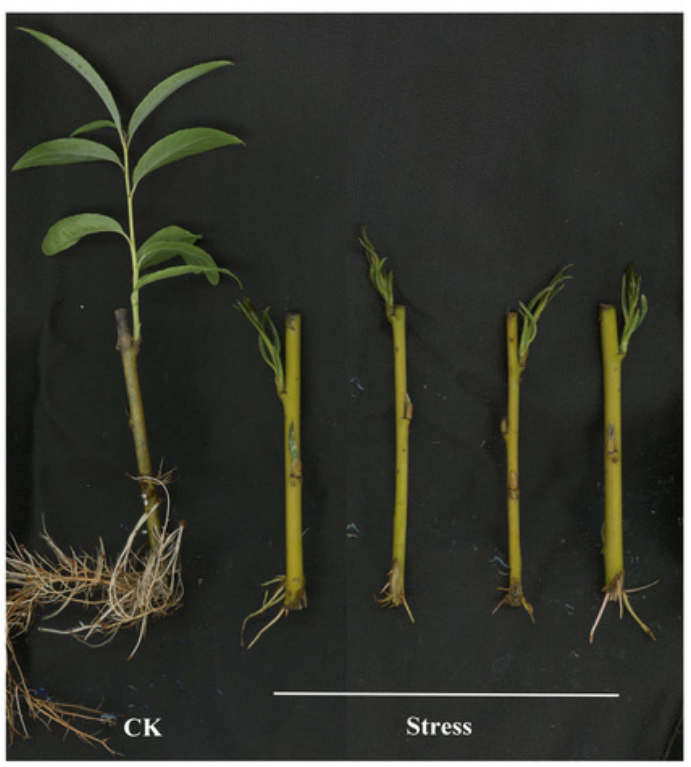

Amount
B

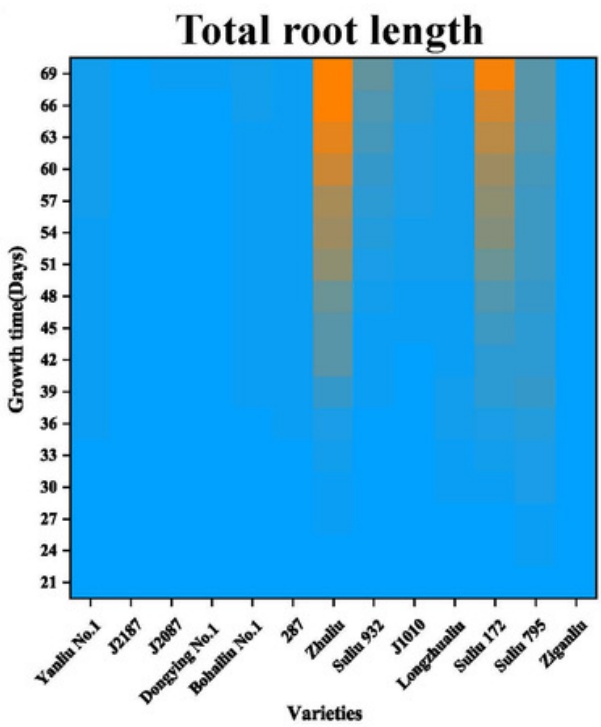

D
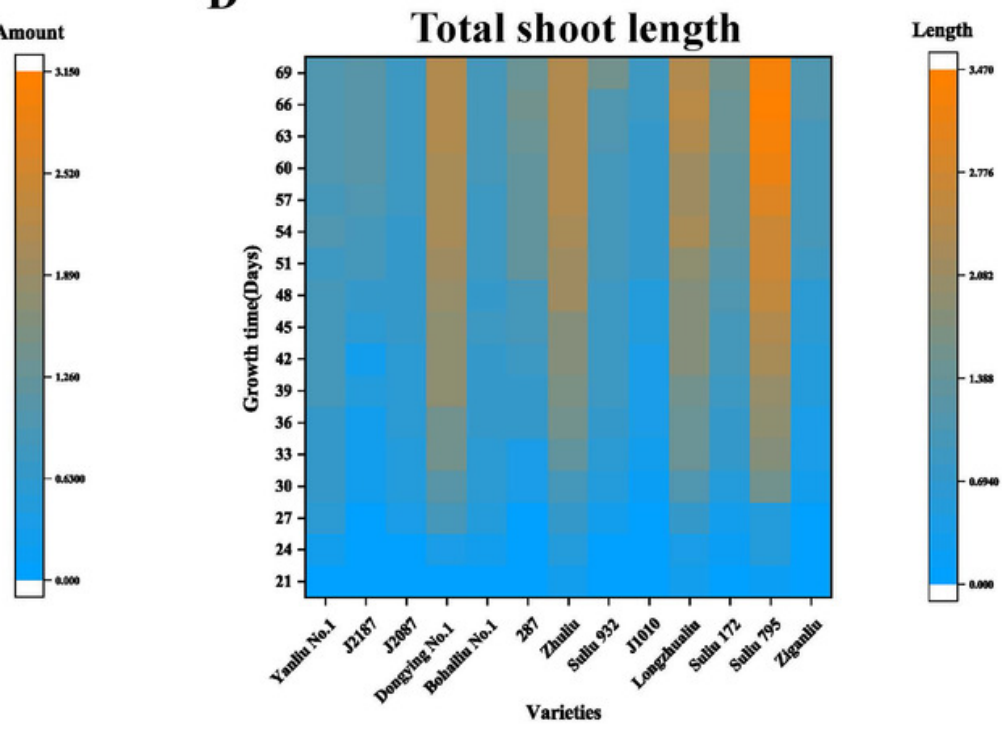

F

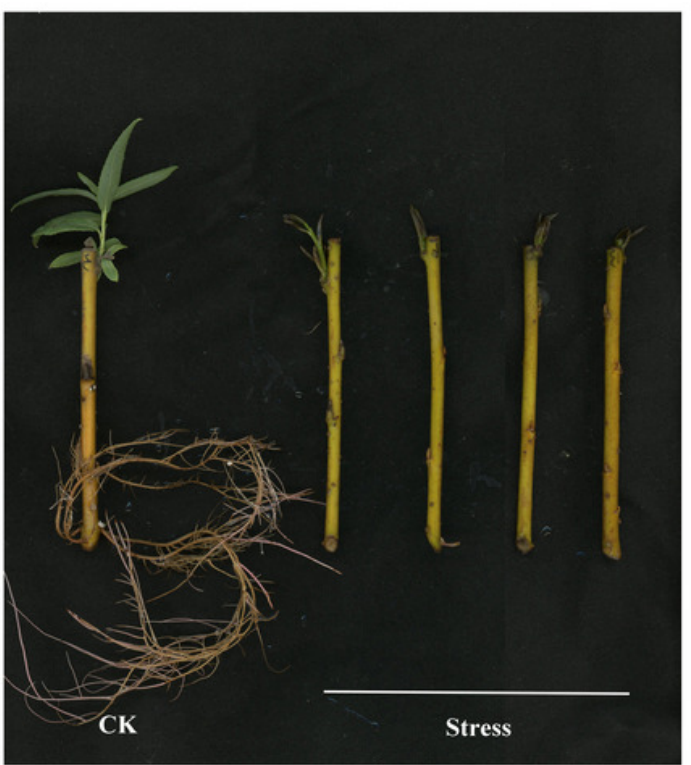


Figure 2

Figure 2: Statistical analysis on RNA sequencing results.

(a) PCA analysis. (b) Statistical analysis of differentially expressed genes (DEGs) from 25

comparisons (5 categories). (c) Venn diagrams of upregulated and downregulated DEGs from the comparisons of categories $\mathrm{A}, \mathrm{B}$, and C. 
A

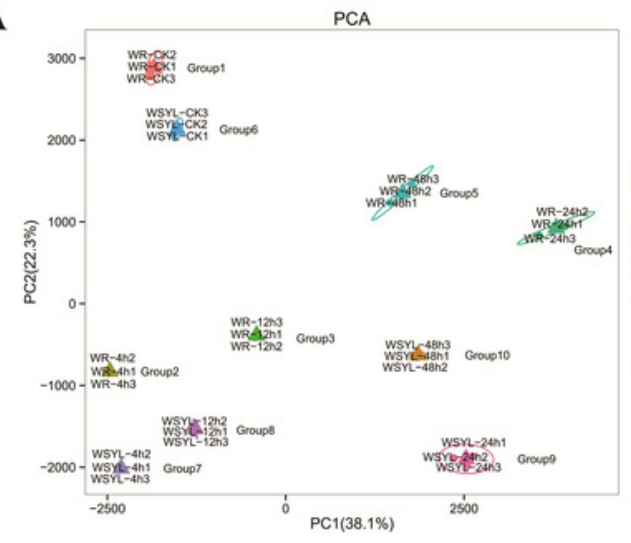

B

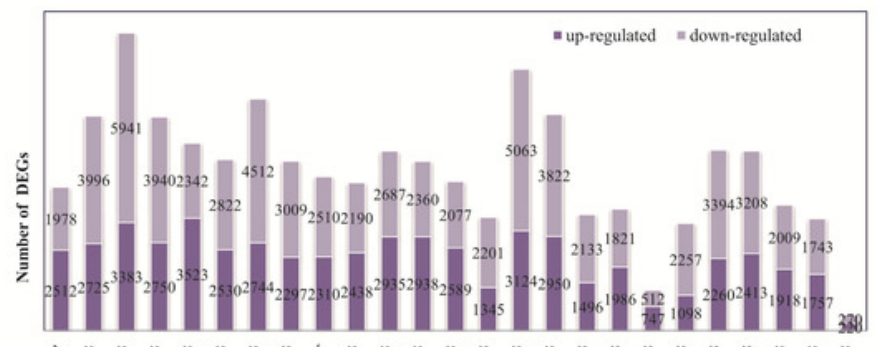

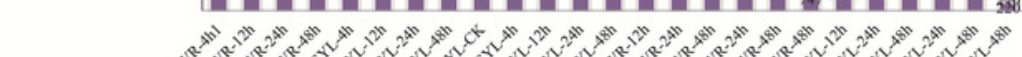
sos Category

C
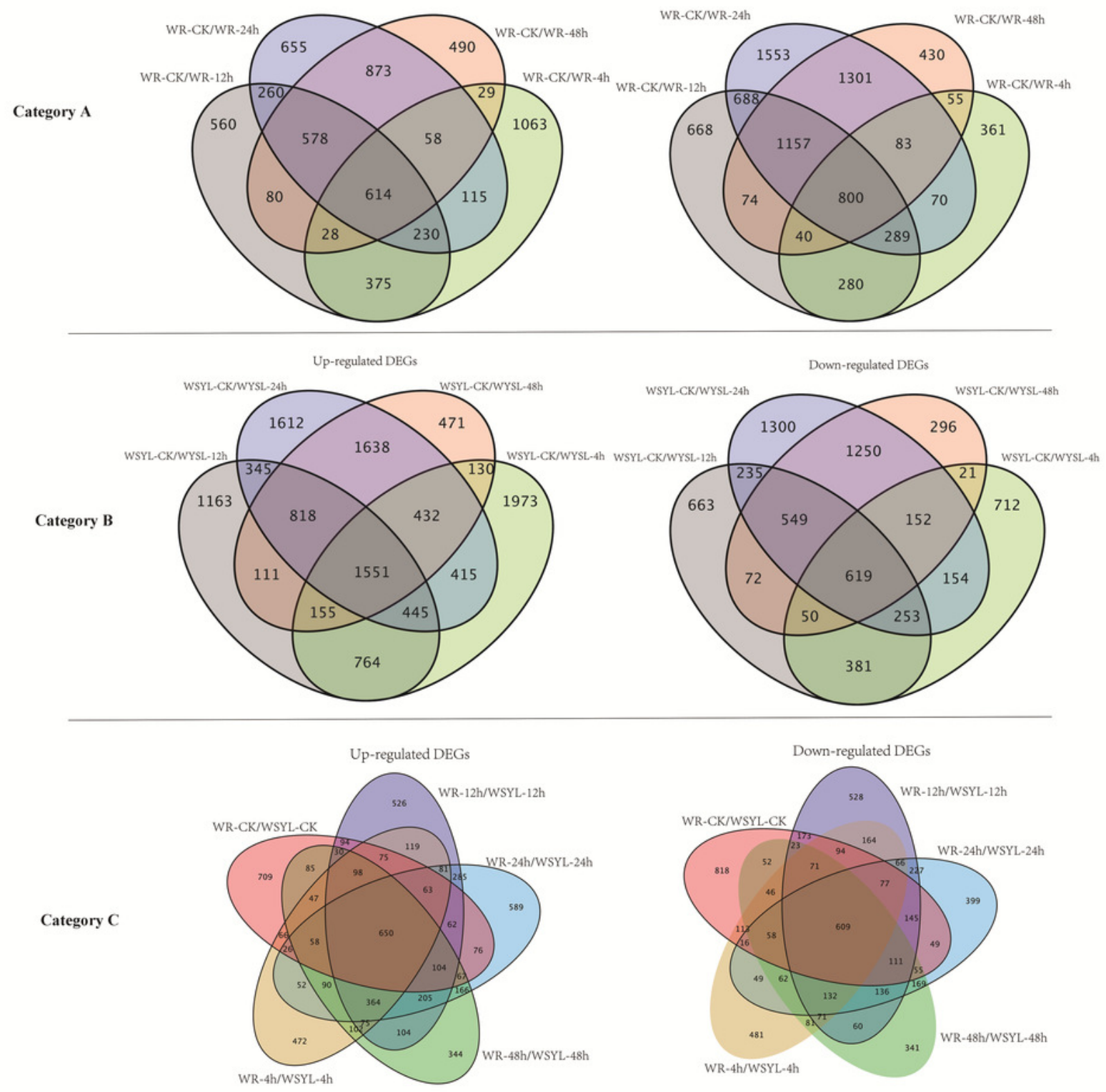


\section{Figure 3}

Figure 3: Biological processes in gene ontology (GO) enrichment analysis of DEGs during submergence stress in the submergence-tolerant and -sensitive varieties of Salix.

Figure 3: Biological processes in gene ontology (GO) enrichment analysis of DEGs during submergence stress in the submergence-tolerant and -sensitive varieties of Salix. GO enrichment analysis was performed using Blast2GO. Only significantly enriched terms (top twenty) with corrected $\mathrm{P}<0.05$ were indicated. The color and size of each point represents the $-\log 10$ (FDR) values and enrichment scores. A higher - $\log 10$ (FDR) value and enrichment score indicate a greater degree of enrichment. Red and blue labeled-terms represent the different and identical terms in different comparisons, respectively. 


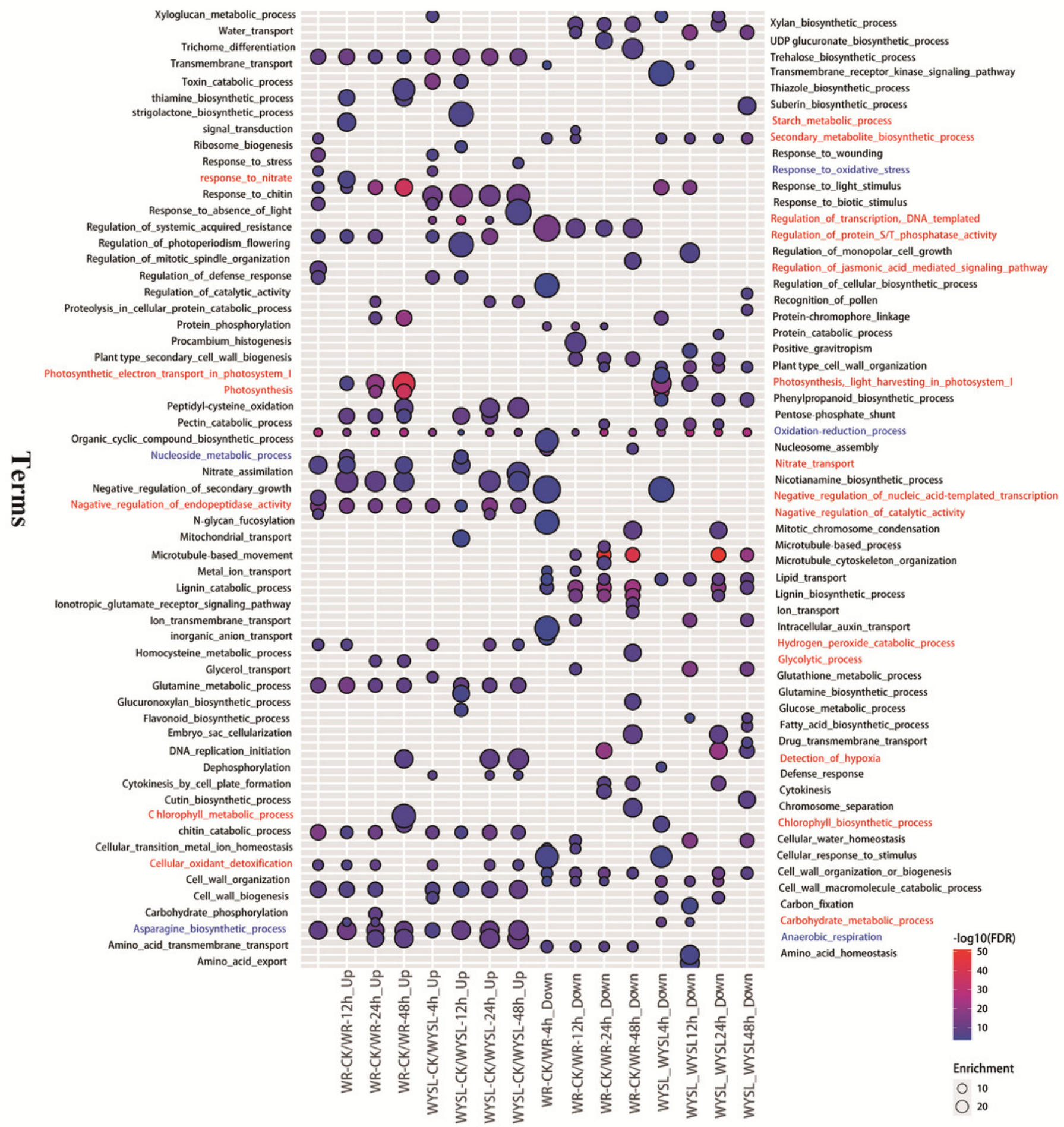




\section{Figure 4}

Figure 4: Statistical analysis and heatmap illustration on TF DEGs.

Figure 4: Statistical analysis and heatmap illustration on TF DEGs. (a) Statistical analysis of transcription factor (TF) differentially expressed genes (DEGs). (b) Heatmap illustration on the expression profiles of some TF DEGs from seven families. (c) Heatmap illustration of the expression profiles of 14 members from the AP2/ERF GroupVII subfamily. 


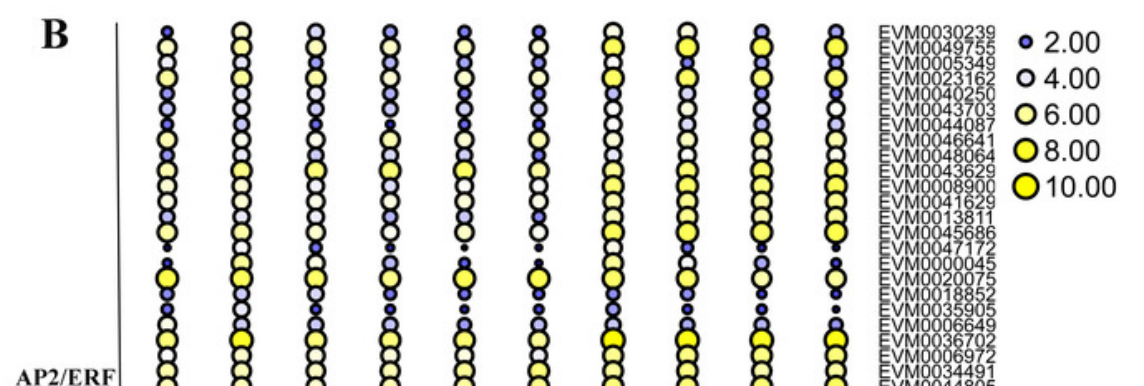

A

C

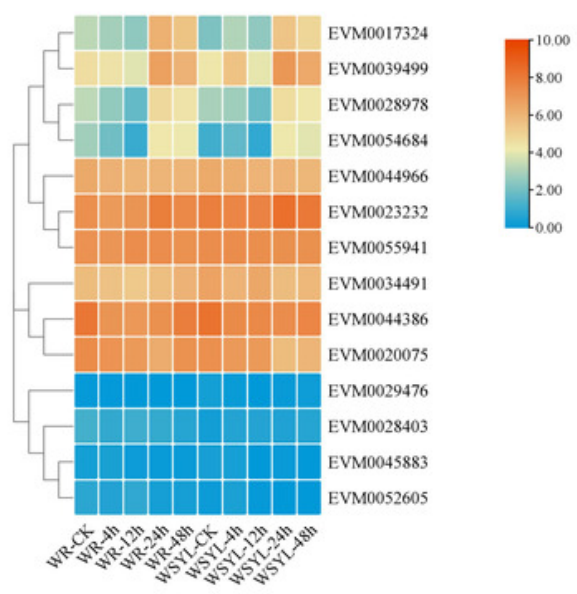

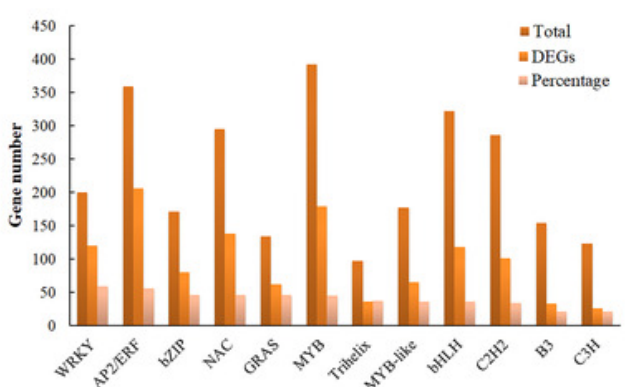

(1)

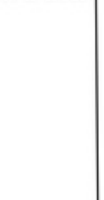

bZIP
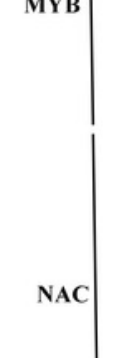

Trihelix

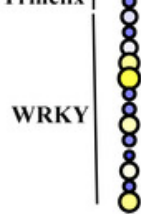

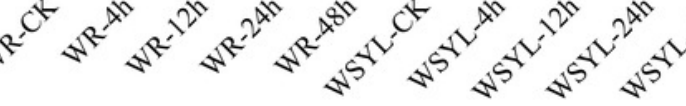

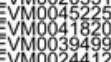

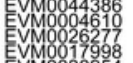

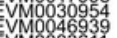

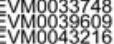

WMo 043770

EMMo

WMo00347

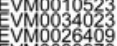

WMo039970

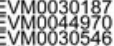

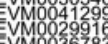

EMoostis

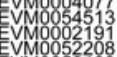
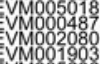

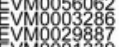

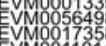

VMooi1014

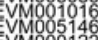


Figure 5

Figure 5: Heatmap analysis of differentially expressed genes (DEGs) encoding for important players in submergence response.

Figure 5: Heatmap analysis of differentially expressed genes (DEGs) encoding for important players in submergence response. (a) Heatmap analysis of differentially expressed genes (DEGs) encoding for important factors in carbohydrate metabolism and anaerobic respiration. (b) ROS signaling pathway and N-end rule pathway. (c) Ethylene, GA, and SA synthesis and signaling.

A

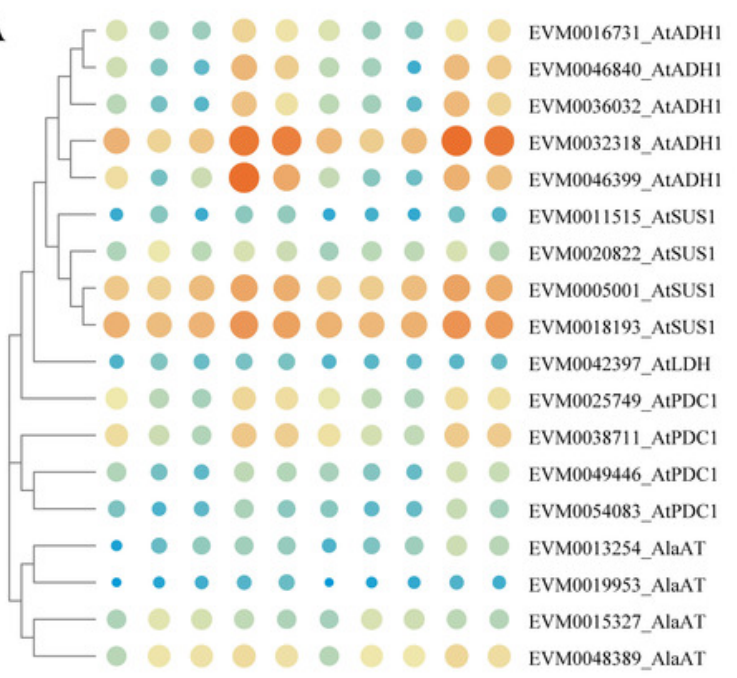

B

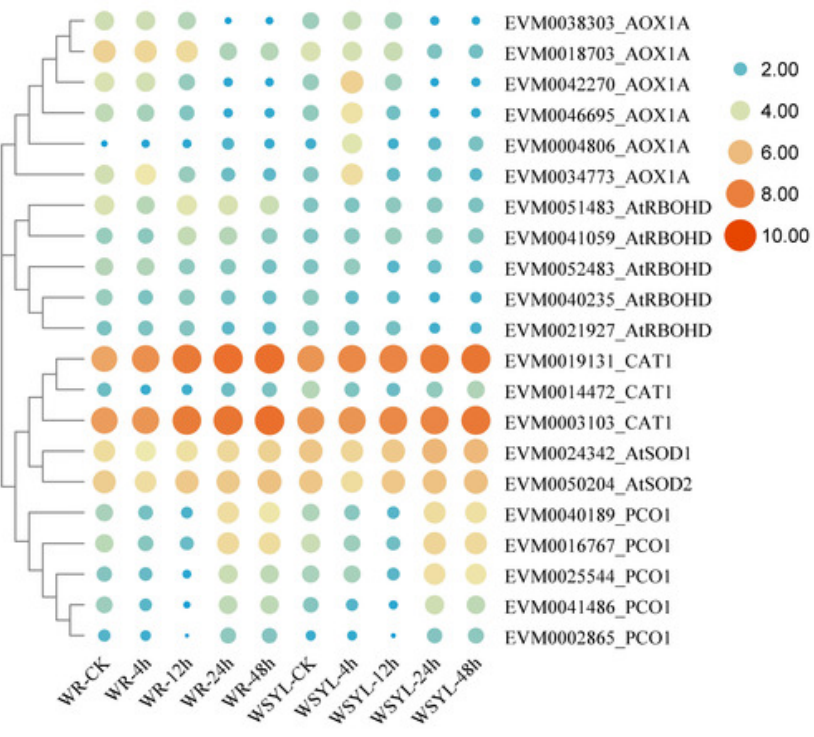

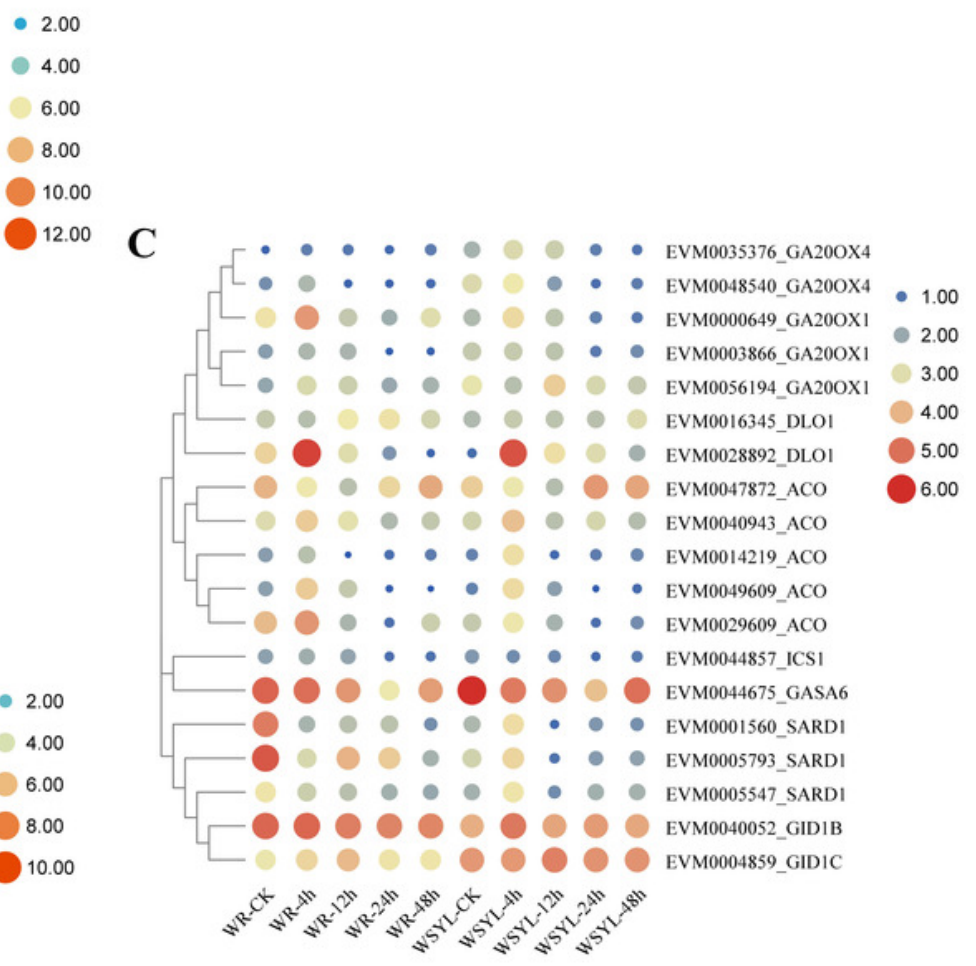

- 2.00

8.00

C

- 10 EVM0005793_SARD

$3 x^{2}+2$ 


\section{Figure 6}

Figure 6: Identification of hub genes in co-expression network under submergence stress.

Figure 6: Identification of hub genes in co-expression network under submergence stress. (a) Eleven hub genes were identified from the red module. (b) Five hub genes were identified from the green module. The co-expression network was analyzed using WGCNA software and the graphic network was created by Cytoscape. 

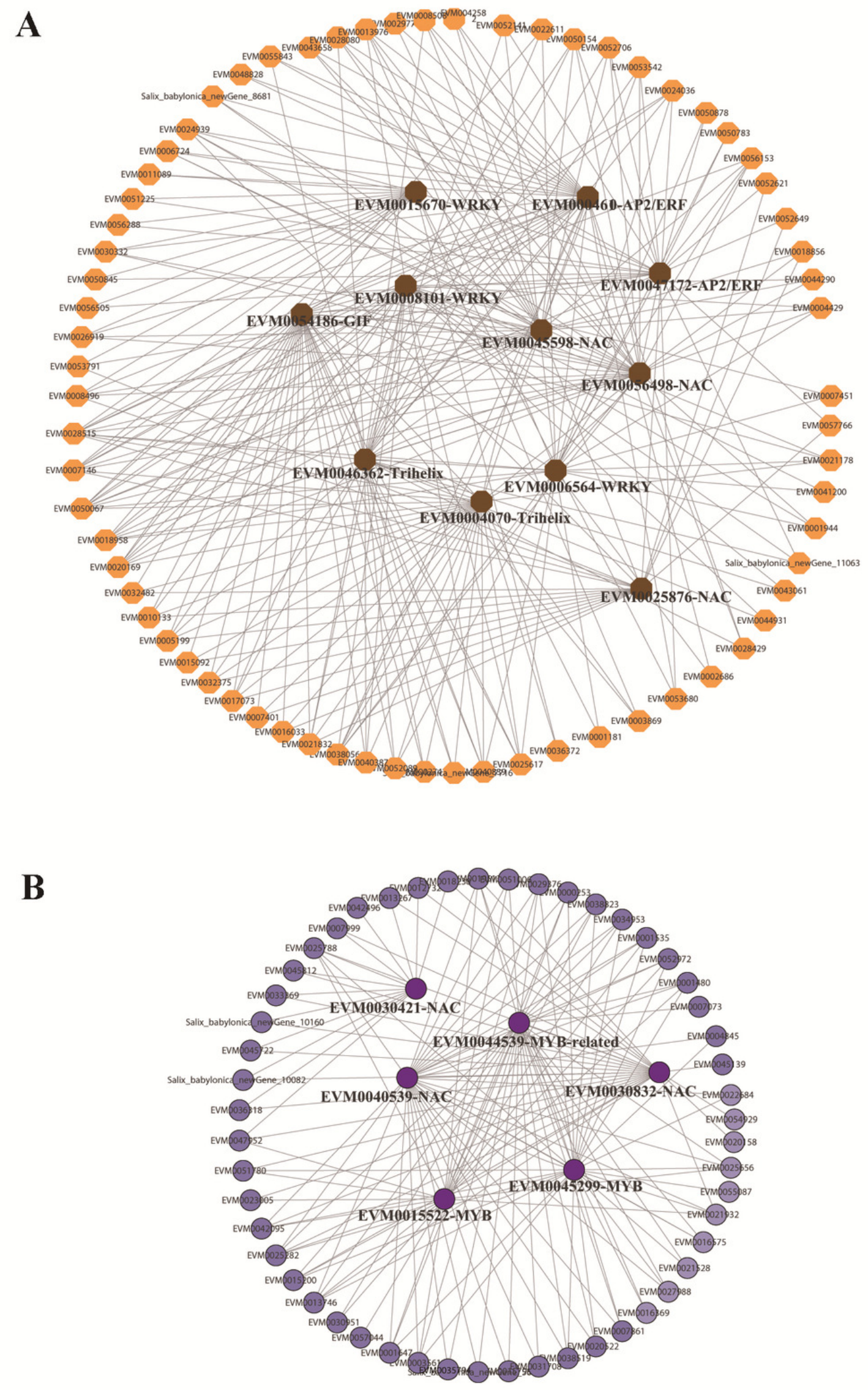
Figure 7

Figure 7: Verification of the DEGs with differentially expressed patterns under submergence stress by quantitative real-time PCR.

Figure 7: Verification of the DEGs with differentially expressed patterns under submergence stress by quantitative real-time PCR. The gene expression profiles were evaluated using the $2^{-\Delta \Delta c t}$ method, and the control values were normalized to 1 . Three biological replicates were performed for each sample. Bars represent the standard deviation of the mean. 

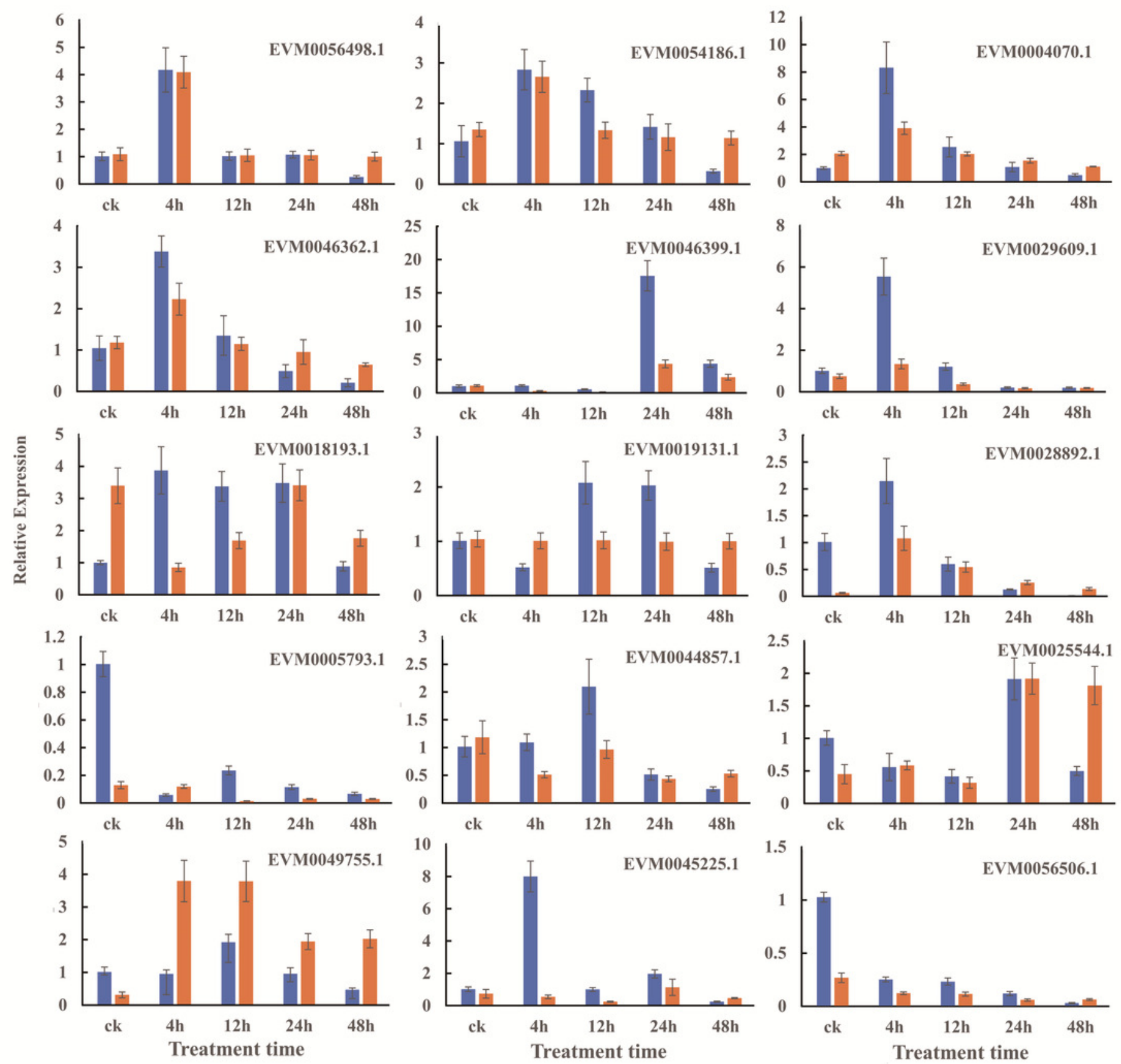

Suliu795

Yanliu No. 1 
Figure 8

Figure 8: The potential mechanisms for Submergence-tolerant willow "Suliu 795" gaining a higher tolerance

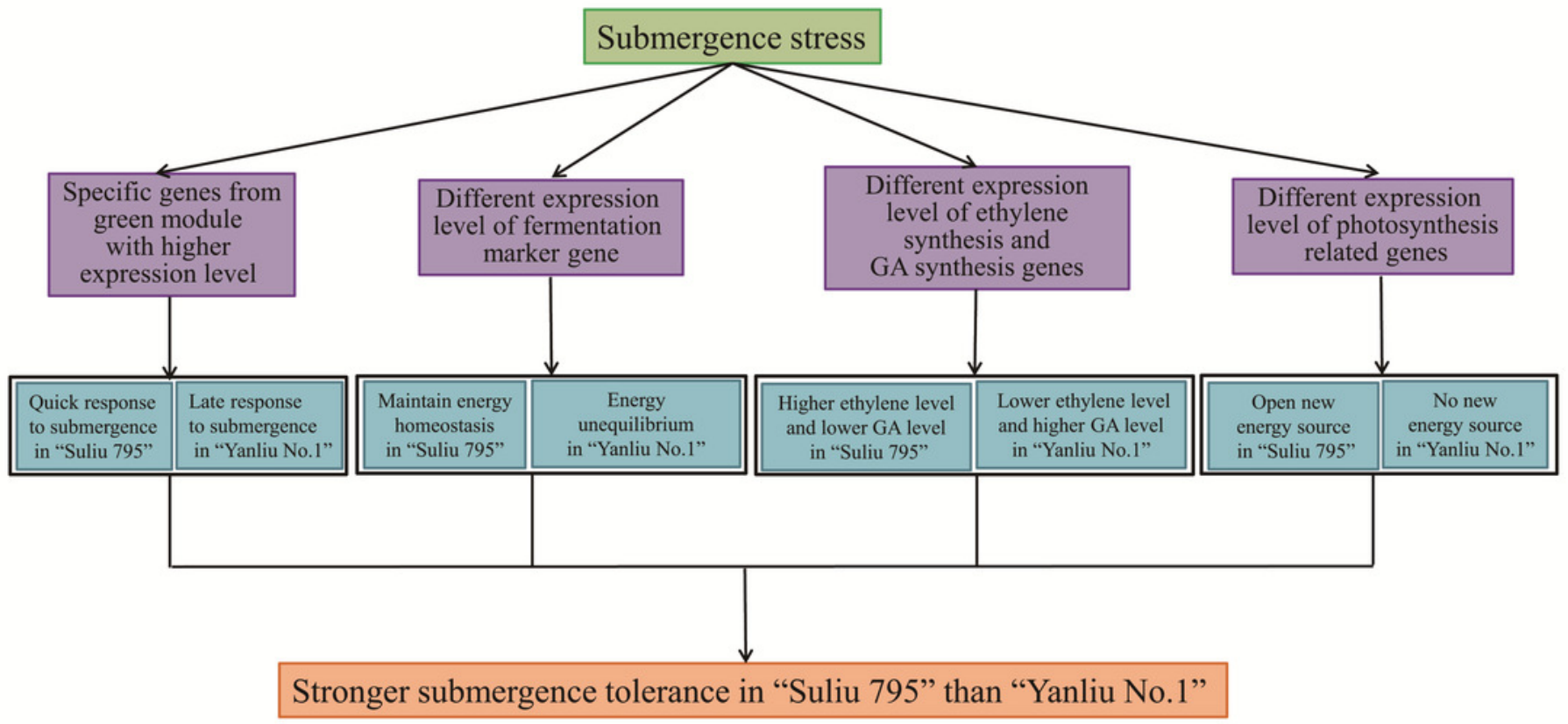

